\title{
Encoding and retrieval of associative recognition memory engage different sub-networks within a hippocampal-thalamo-cortical memory circuit
}

\section{Gareth Barker}

https://orcid.org/0000-0002-0918-2045

\section{Stephanie Tran}

University of Bristol https://orcid.org/0000-0002-0036-5195

\section{Kerry Gilroy}

University of Bristol

\section{Zafar Bashir}

University of Bristol

Elizabeth Warburton ( $D$ E.C.Warburton@bristol.ac.uk )

University of Bristol

\section{Article}

Keywords: memory encoding, memory retrieval phases, associative recognition memory, hippocampalthalamo-cortical memory circuit

Posted Date: October 1st, 2021

DOI: https://doi.org/10.21203/rs.3.rs-889826/v1

License: (c) (i) This work is licensed under a Creative Commons Attribution 4.0 International License. Read Full License 


\section{Encoding and retrieval of associative recognition memory engage different sub-networks within a hippocampal-thalamo-cortical memory circuit}

Gareth R.I. Barker, Stephanie Tran, Kerry Gilroy, Zafar I. Bashir and E. Clea. Warburton*

School of Physiology, Pharmacology and Neuroscience, University of Bristol, Bristol BS8 1TD, U.K.

*corresponding author

Recognition of previously encountered stimuli and their associated spatial and temporal information depends on neural activity within a brain-wide network in which the CA1 region of the hippocampus, nucleus reuniens of the thalamus (NRe) and medial prefrontal cortex (mPFC) are key nodes. However, the pathways crucial for coordinating activity during memory encoding and/or retrieval phases have been little explored. Here we opto- or chemo associative recognition memory. We discovered that encoding, but not retrieval depended on the CA1 to MPFC and NRe to MPFC projections. In contrast, retrieval depended on the MPFC to NRe projection. Interestingly the NRe to CA1 pathway was required for both memory phases. Our findings therefore reveal that encoding and retrieval engage dissociable sub-networks within a hippocampal-thalamo-cortical recognition memory circuit in order to enable binding of recent and related information, whilst ensuring a separation of processing. 
Remembering individual stimuli with associated spatial and temporal information is crucial for successful episodic memory, can facilitate effective recognition and guide future behaviour. Both spatial (object-place) and temporal order memory are characterised by coordinated neural activity in the hippocampus (HPC) and medial prefrontal cortex (MPFC) $(1 ; 2 ; 3 ; 4 ; 5)$. Studies have also shown that temporary lesions in these regions significantly impair encoding and retrieval of both forms of memory $(6 ; 7 ; 8)$. Recently, the nucleus reuniens of the thalamus (NRe) which is heavily interconnected with the HPC and MPFC $(9 ; 10 ; 11 ; 12 ; 13 ; 14)$ has been shown to be crucial for both encoding and retrieval of spatial and temporal recognition memory $(8 ; 15)$. Finally, disconnection analyses indicate that pairs of these structures appear to work in concert $(16 ; 8)$, suggesting the existence of a complex HPC-NRe-mPFC memory network. A key question however concerns which specific neural pathways are involved in the relay of information across the circuit.

Studies of the cellular correlates of memory suggest encoding involves the activation and consequent synaptic modifications of distinct populations of neurons creating an 'engram' $(17 ; 18)$ while memory retrieval involves the fast reactivation of the engram (19).

Theoretically therefore, encoding and retrieval could be achieved through reactivation of the same neural pathways. However, as retrieval is initiated by an external or internal cue, there must be interactions between the cue and the stored engram with the additional influence of top-down monitoring processes. Encoding and retrieval are likely, therefore to involve the activation of different neural pathways. Indeed, electrophysiological studies indicate that contextual memory encoding may be driven by HPC inputs to the MPFC while retrieval may be mediated by mPFC output pathways $(1 ; 2)$. Such findings point to the intriguing possibility that associative memory encoding and retrieval may be supported by 
functionally distinct sub-networks within the HPC-NRe-mPFC memory circuit, yet this hypothesis has yet to be systematically tested.

To examine the role of HPC-mPFC-NRe pathways in associative memory we used optogenetic and chemogenetic manipulations to inactivate specific neural pathways during the encoding or retrieval phases of an associative object-in-place or object temporal order recognition memory task. These tasks which rely on an animal's innate preference for novelty, map more closely onto human declarative memory than other rodent tests of memory and as they are one-trial tests they are suitable for dissociating encoding from retrieval events $(20 ; 21 ; 22)$. Here we found that encoding, but not retrieval required inactivation of $\mathrm{CA} 1 \rightarrow \mathrm{mPFC}$ and $\mathrm{NRe} \rightarrow \mathrm{mPFC}$ projections, while retrieval selectively depend on the $\mathrm{mPFC} \rightarrow \mathrm{NRe}$ projection. Interestingly, the NRe $\rightarrow$ CA1 projection was involved in both phases of memory. Importantly we demonstrated a clear dissociation between the involvement of these pathways when animals were required to both encode and retrieve item information simultaneously. These results reveal the existence of distinct neural networks for encoding and retrieval of associative recognition memory information and thus provide clear and novel insights into the operation of brain-wide memory circuitry.

\section{RESULTS}

\section{Optogenetic inactivation of a specific anatomical projection decreases c-fos expression}

To disrupt neuronal activity in the CA1-mPFC-NRe network, pathway specific optogenetic and chemogenetic manipulations were used. Viral vectors expressing either inhibitory opsins or Designer Receptors Exclusively Activated by Designer Drugs (DREADDs) were injected into the projection area and optrodes or cannulae were targeted to the axonal output (Fig 1a, 2a;c, 4a). Thus, laser light delivery or clozapine-N-oxide (CNO) infusion 
should selectively deactivate the projections from the site of viral injection to the site of optrode/cannulae implantation. The decision on whether to use optogenetic or chemogenetic depended on the size of the target region, thus DREADD inactivation was used in to deactivate the inputs, from NRe into the dorsal or intermediate CA1 region of the hippocampus (dCA1 or iCA1).

To confirm that light delivery attenuated neuronal activation in the target region, we measured c-fos expression levels in MPFC after deactivation of the NRe $\rightarrow$ mPFC projection. In each animal an AAV vector expressing either AAV5-CamKII-eARCH3.0-EYFP or AAV5CamKII-EYFP as a control (hereafter called ARCH or YFP) was injected into the NRe and optrodes were implanted bilaterally into the mPFC. Animals were placed in the behavioural arena which contained four different objects (as in the sample phase of the object-in-place task) and received unilateral laser stimulation. Ninety minutes later the animals were sacrificed and c-fos levels in mPFC were measured by immunohistochemistry. Expression of c-fos was compared within each animal between the hemisphere which received laser light delivery and the side which did not receive laser light delivery (Supplementary Data Fig 1a). It was found that laser stimulation of $\mathrm{NRe} \rightarrow \mathrm{mPFC}$ projection significantly decreased c-fos expression in the mPFC (raw data counts Supplementary Data Fig 1b; normalised counts $t(11)=-6.07, P=0.0001$, Supplementary Data Fig $1 c)$, in contrast laser light delivery did not significantly alter c-fos expression in animals expressing the control vector (YFP) (raw data counts Supplementary Data Fig $1 \mathrm{~b}$; normalised counts $\mathrm{t}(11)=1.01, \mathrm{P}=0.335$, Supplementary Data Fig 1c). 


\section{Inactivation of the direct intermediate CA1->MPFC projection impairs encoding but not retrieval of object-in-place memory}

We have previously shown that long-term inactivation of the intermediate CA1 (iCA1) to the mPFC projection impairs object-in-place memory, but this study did not address whether the pathway was involved during encoding or retrieval or both (7). To answer this question, here we used an optogenetic approach to transiently inactivate the direct iCA $1 \rightarrow \mathrm{mPFC}$ projection either during the sample phase, so as to affect encoding or during the test phase, so as to affect retrieval. Rats received bilateral injections of ARCH or YFP into the iCA1 region and optrodes were implanted bilaterally into the mPFC so as to deliver light stimulation to the terminal regions of the iCA1 projection (Fig. 1a;b). To assess object-inplace memory, rats were placed in the arena and allowed to explore four different objects placed in specific locations within the area. The test phase occurred after a $5 \mathrm{~min}$ or $3 \mathrm{~h}$ retention delay. At test rats were placed back in the arena in which two of the objects were in the same location as in the sample phase, while two objects had exchanged positions. Exploration of all four objects was measured and object-in-place memory considered intact if, the animal preferentially explored the objects in different locations to that of the sample phase (Fig. 1c). Analysis of the behaviour in the test phase, following either delay period, showed that when light stimulation was applied selectively during the sample phase, the YFP animals spent a greater proportion of time exploring the moved compared to the unmoved objects, indicating no effect memory performance. In contrast the ARCH animals spent an equal amount of time exploring the moved and unmoved objects, indicating impaired memory performance ( $3 \mathrm{~h}$ delay: virus by laser interaction $F(1,17)=19.07, P=0.001$, Fig. 1d, 5 min delay: Supplementary Data Figs. 2a;b). In the next experiment object-in-place memory was tested again in the same animals (seven days later) using new objects but with 
light stimulation applied selectively during the test phase (Fig. 1e). Now both the YFP-and ARCH-treated animals spent longer exploring the moved compared to the unmoved objects (3h delay: virus by laser interaction $F(1,18)=0.11, P=0.743$, Fig. $1 f$, 5 min delay: Supplementary data Figs. $2 c ; d$ ). Thus optogenetic inhibition of the iCA1->mPFC pathway during the test phase, had no detrimental effect on retrieval performance. Total object exploration in either phase of the procedure did not significantly differ between either virus (ARCH/YFP) or laser condition (On/Off ; see Table 1). Together these results show that activity in the $i C A 1 \rightarrow m P F C$ projection is specifically required during encoding, but not during retrieval of object in place memory.

To demonstrate the functional specificity of this dissociation between encoding and retrieval, we next tested the animals in a temporal order memory task previously shown not to depend on $i C A 1 \rightarrow m P F C$ projections (7). In this task (Fig. 1g) animals are presented with a series of objects in four sample phases (S1-S4). At test, one object from S2 and one object from S3 are presented simultaneously and memory is considered intact if the animal preferentially explores the object which appeared earlier in the sequence, i.e object from S2. Here, as predicted, application of light stimulation during S3 (to affect encoding) had no effect on temporal order memory performance in the ARCH or YFP groups (virus by laser interaction $\mathrm{F}(1,18)=0.67, \mathrm{P}=0.743$, Fig. $1 \mathrm{~h})$. Finally, deactivation of the $i C A 1 \rightarrow \mathrm{mPFC}$ projection also had no effect on performance of a hippocampus-independent object recognition memory task (Supplementary Data Fig. 2e;f).

Double dissociation in the role of NRe->MPFC and mPFC->NRe projections in memory encoding and retrieval 
Considering the evidence that the NRe is involved in associative memory ( 8 and see Supplementary Data Figure 3a;b), we next examined the effect of optogenetic inhibition of the $\mathrm{NRe} \rightarrow \mathrm{mPFC}$, and $\mathrm{mPFC} \rightarrow$ NRe pathways on object-in-place memory. Thus to manipulate the NRe $\rightarrow$ mPFC pathway AAV5-CamKII-eARCH3.0-EYFP or AAV5-CamKII-EYFP viruses were injected bilaterally into the NRe and optrodes were implanted to enable light into the mPFC (Fig 2a;c). To manipulate the reciprocal (mPFC $\rightarrow$ NRe) pathway AAV5-CamKIIeARCH3.0-EYFP or AAV5-CamKII-EYFP viruses were injected into the mPFC and optrodes were implanted into the NRe (Fig. 2b;d). As before animals were tested in the object-inplace task (Fig. 1c\&d) with light applied either during the sample (to affect encoding) or during the test phase (to affect retrieval).

Inhibition of the $\mathrm{NRe} \rightarrow \mathrm{mPFC}$ projection terminals during the sample phase significantly impaired performance in the ARCH animals while inhibition of the NRe $\rightarrow$ mPFC projection terminals during the test phase had no effect (Fig. 2e;f). In contrast inhibition of the $\mathrm{mPFC} \rightarrow$ NRe projection terminals during the sample phase had no effect, while inhibition during the test phase significantly impaired memory (Fig. $2 \mathrm{~g} ; \mathrm{h}$ ). These results suggest that object-in-place memory encoding depends on the activation of the $\mathrm{NRe} \rightarrow \mathrm{mPFC}$ while retrieval requires activation of the $\mathrm{mPFC} \rightarrow \mathrm{NRe}$ pathway.

There are two possible explanations for the retrieval impairment following inactivation of the NRe projecting mPFC cells. Firstly, the deficit could reflect an erasure of encoded information or secondly, it could reflect the animal's temporary inability to access the stored object-place representations. To examine these possibilities, we re-tested the $\mathrm{mPFC} \rightarrow \mathrm{NRe}$ animals in the object-in-place task, this time using a 6 minute test phase, in which the light was delivered to the terminals in the NRe during the first 3 minutes only 
(Supplementary Data Fig. 4a). As expected in the first 3 minutes, memory was significantly impaired, however when the light was switched off animals showed intact memory, thus indicating that the manipulation had not resulted in a loss of encoded information (threeway ANOVA $F(1,22)=14.62, P=0.001$, Supplementary Data Fig. 4b;c;d;e). Instead, this result suggests that the mPFC $\rightarrow$ NRe projection is required for access to encoded object-place associations for successful memory retrieval.

The observation that it was possible to doubly dissociate the effects of inhibition of the $\mathrm{NRe} \rightarrow \mathrm{mPFC}$ and $\mathrm{mPFC} \rightarrow \mathrm{NRe}$ pathways on encoding and retrieval was confirmed using the temporal order memory task. Here light stimulation was applied either during sample phase 3 (S3), to affect encoding or during the test phase, to affect retrieval (Fig. 2i;j). Again, inhibition of $\mathrm{NRe} \rightarrow \mathrm{mPFC}$ projection terminals during S3, but not during the test phase impaired performance (Fig. 2l;j). Conversely inhibition of $\mathrm{mPFC} \rightarrow \mathrm{NRe}$ during the test phase, but not during the sample phase significantly impaired performance (Fig. $2 k ; l)$. Optogenetic manipulation of either pathway did not significantly alter total object exploration levels in the sample or test phases (Table 2).

Previous studies have shown the NRe to be critical for long-term but not short-term objectin-place memory (8) and here we found that deactivation of the either the mPFC $\rightarrow \mathrm{NRe}$ projection during memory encoding or the $\mathrm{NRe} \rightarrow \mathrm{mPFC}$ projection during memory retrieval did not alter performance in the object-in-place task with a short delay (Supplementary Data Fig. 3c-f).

To test the functional specificity of the observed effects, we next tested novel object recognition memory. Deactivation of the $\mathrm{mPFC} \rightarrow \mathrm{NRe}$ projection terminals during the 
sample phase of the novel object recognition memory task, or the NRe $\rightarrow \mathrm{mPFC}$ during the test phase did not significantly alter performance (Supplementary Data Fig. 3g-j).

Together these results demonstrated that the selective necessity of the NRe $\rightarrow$ mPFC pathway during the encoding phase of object-in-place and temporal order tasks, and the necessity of the mPFC $\rightarrow$ NRe pathway for retrieval of such information.

\section{NRe-mPFC interactions dynamically regulate memory encoding and retrieval}

The acquisition of new information during learning rarely, if ever, occurs in isolation from ongoing information retrieval. A key question, therefore, is whether it is possible to dissociate the anatomical basis of encoding and retrieval when they occur simultaneously. Given that we have demonstrated that encoding and retrieval of object-in-place information are mediated by the NRe $\rightarrow \mathrm{mPFC}$ and $\mathrm{mPFC} \rightarrow \mathrm{NRe}$ pathways respectively, it should be possible to manipulate these processes independently during the same phase of a single task. To test this assumption, we modified the object-in-place task to include two test sessions (Fig 3a). By using two test session we can examine whether it is possible to block encoding of new information while still observe the retrieval of previously learnt information in test phase 1 , or conversely block retrieval yet enable encoding of new information, and thus observe the double dissociation of these two processes. In this new version of the object-in-place task the animals, underwent a standard sample phase in which they explored four objects, $A, B, C, D$ followed 3 hours later by the first test phase (test phase 1 ) in which two objects ( $C \& D$ ) exchange position, and objects $A$ and $B$ remain in the same position. Thus, during test phase 1 control animals should be able to both retrieve the object-place associations formed in the sample phase and encode the new object-place 
associations created by the moved objects. Three hours after test phase 1 the animals were tested in test phase 2 , in which objects $A$ and $B$ exchanged position, while objects $C$ and $D$ remained in the same position as in test phase 1 . Light stimulation was applied to all groups during test phase 1 only (Fig. 3a).

Memory performance in the NRe $\rightarrow$ mPFC and $m P F C \rightarrow$ NRe groups was examined in test phases 1 and 2. In the NRe $\rightarrow$ mPFC group, light application had no effect on performance in the ARCH- or YFP-treated animals (three-way ANOVA $F(1,22)=13.49, P=0.001$, Fig. 3b). In test phase 2 , however memory performance in the $\mathrm{ARCH}$ animals was significantly impaired (Fig 3b). In contrast manipulation of the $\mathrm{mPFC} \rightarrow$ NRe pathway produced a different pattern of results. Thus, in test phase 1 , object discrimination was impaired in the $\mathrm{ARCH}$-treated animals while the YFP animals showed significant discrimination (three-way ANOVA $F(1,22)=15.92, P=0.001$, Fig. $3 c)$, while in test phase 2 all groups were able to discriminate between the moved and unmoved objects, i.e. memory performance was intact (Fig 3c). Total object exploration levels, in the sample phase, test phase 1 or 2 did not vary between conditions (Table 3).

These results confirmed that it is possible to selectively impair either memory encoding or retrieval when both processes are occurring simultaneously.

Retrieval, but not encoding of object-in-place and temporal order memory require the integrity of task specific parallel NRe $\rightarrow$ CA1 pathways.

As previously shown, the $d \mathrm{CA} 1 \rightarrow \mathrm{mPFC}$ and $i \mathrm{CA} 1 \rightarrow \mathrm{mPFC}$ pathways mediate temporal order and object-in-place memory respectively (7). The NRe projects across the dorso-ventral 
extent of the hippocampus (23), hence the next experiment tested whether the mnemonic role of the NRe projections to the CA1 could be functionally dissociated. Due to the volume of the hippocampal regions that we wished to manipulate, this experiment used a DREADDbased chemogenetic, rather than optogenetic approach to manipulate separately the $\mathrm{NRe} \rightarrow d \mathrm{CA} 1$ and $\mathrm{NRe} \rightarrow i \mathrm{CA} 1$ pathways. A viral vector expressing the inhibitory DREADD AAV5-hSyn-hM4Di-mCherry was injected into the NRe and bilateral guide cannulae were implanted targeting both the $d C A 1$ and $i C A 1$ region of the hippocampus to allow for the intracerebral infusion of clozapine $N$-oxide (CNO), into the $d C A 1$, iCA1 or simultaneously into both regions (Fig 4a-d). CNO or vehicle infusions were made into the regions according to a Latin square design either before the sample phase, to test for effects on encoding, or before the test phase, to test for effects on retrieval of the object-in-place or temporal order memory task (Fig. 4e-h).

Infusions of $\mathrm{CNO}$ into either the $d C A 1$ or iCA1, prior to the sample phase had no impact on encoding of object-in-place memory. However, simultaneous infusions into both regions before the sample significantly impaired memory performance (one-way ANOVA $F(3,42)=20.80, P=0.001$, Fig. 4 e). A similar pattern of results was found in the temporal order memory task, where administration of CNO only impaired encoding when co-infused into $d C A 1$ and $i C A 1$, but not when infusions were made into either region alone (one-way ANOVA $F(3,42)=26.04, P=0.001$, Fig. $4 \mathrm{~g})$. Interestingly a different pattern of results was observed in both the object-in-place and temporal order tasks when CNO was administered prior to the test phase. Here infusion of $\mathrm{CNO}$ into the iCA1, but not into $d \mathrm{CA} 1$ significantly impaired object-in-place memory (one-way ANOVA $F(3,42)=38.01, P=0.001$, Fig. 4f), and infusion of CNO into the $d C A 1$, but not the iCA1 impaired temporal order memory (one-way ANOVA $F(3,42)=25.92, P=0.001$, Fig. 4h). Not surprisingly co-infusion of CNO into both 
hippocampal subregions, prior to test impaired performance in both tasks (Fig. 4f;4h).

Infusion of CNO did significantly alter overall object exploration in some experiments (Table 4), however none of the groups which showed impaired memory performance had significantly lower or higher exploration levels compared to animals which received a vehicle infusion.

Finally, to investigate the selectively of the role of the $\mathrm{NRe} \rightarrow \mathrm{CA} 1$ pathway we examined short term object-in-place memory, object recognition and object location memory, as previously studies have shown that NRe is not required for performance of these tasks. Results showed that co-infusion of CNO into both the $d C A 1$ and iCA1 did not alter performance in either the object-in-place task tested with a short delay $(5 \mathrm{~min})$ (Supplementary Data Figure 5a;b) or performance in the object location or object recognition task (Supplementary Data Figure 5c-f).

These results indicate that the $\mathrm{NRe} \rightarrow \mathrm{CA} 1$ projection is involved in both encoding and retrieval of associative recognition, but, depending on the precise nature of the memory information, retrieval recruits this pathway in a regionally specific manner.

\section{DISCUSSION}

It is recognised that associative memory depends on hippocampo-thalamo-cortical interactions, yet the roles played by specific circuit projections during encoding and retrieval are unknown. Here this question was addressed by optogenetic or DREADD inactivation of neural pathways between the HPC, MPFC and NRe at distinct phases of object-in-place and temporal order recognition memory tasks. First, encoding of object-in-place memory was 
impaired by inactivation of the iCA1 $\rightarrow$ mPFC pathway, and encoding of both object-in-place and temporal order memory was impaired by inactivation of the NRe $\rightarrow$ mPFC pathway and by simultaneous inactivation of both the NRe $\rightarrow d$ CA1 and NRe $\rightarrow$ iCA1 pathways. In contrast, retrieval of object-in-place and temporal order memory was disrupted by inhibition of the mPFC $\rightarrow$ NRe pathway or the NRe $\rightarrow$ iCA1 (for object-in-place) and NRe $\rightarrow$ dCA1 (for temporal order) pathways. None of the pathway manipulations disrupted novel object recognition or spatial memory of an individual object, meaning that the performance deficits observed could not be accounted for by nonspecific effects of viral transduction, light delivery or infusion of $\mathrm{CNO}$. These results reveal, for the first time, the engagement of different neural pathways during associative memory performance. The specific pathways engaged thus depends on whether information is being encoding or retrieved, and the type of recognition memory information being processed i.e. object-in-place or object-temporal order information.

Encoding, but not retrieval of object-in-place memory required activation of the iCA1 $\rightarrow$ mPFC pathway as well as direct NRe input to both the CA1 and mPFC. Firstly, these results are consistent with studies showing that object-spatial learning is associated with HPC-mPFC neuronal firing synchrony $(24 ; 25 ; 26)$ and that object-place cells appear to be set up in the $\mathrm{HPC}$ and subsequently in the mPFC $(2 ; 27 ; 28)$. While the $i \mathrm{CA} 1 \rightarrow \mathrm{mPFC}$ pathway was required for the encoding of both shorter ( $5 \mathrm{~min}$ ) and long term ( $3 \mathrm{~h})$ memory, the NRe $\rightarrow$ CA1 and $\mathrm{NRe} \rightarrow \mathrm{mPFC}$ projections were only required for long term memory, which raises the question of the role of the NRe input? Studies have shown that projections from the NRe and CA1 converge in layer $\mathrm{V}$ of the mPFC, $(9 ; 29 ; 30)$ and recently we have demonstrated that associative, NMDA receptor dependent synaptic plasticity at both the CA $1 \rightarrow \mathrm{mPFC}$ and $\mathrm{NRe} \rightarrow \mathrm{mPFC}$ synapses is dependent on paired stimulation of the HPC followed by NRe at 
theta frequency (30). Together these behavioural, anatomical and electrophysiological data suggest that during memory encoding, NRe input to the MPFC facilitates plasticity of the CA1-mPFC synapse. Such plasticity is required for the establishment of object-place representations to support longer term memory formation. Over short retention delays, however, memory performance is supported by activity in the direct CA1 $\rightarrow$ mPFC pathway only, and does not require input from the NRe.

As mentioned previously object temporal order and object-spatial memory are mediated by the $d \mathrm{CA} 1 \rightarrow \mathrm{mPFC}$ and $\mathrm{iCA} 1 \rightarrow \mathrm{mPFC}$ projections respectively (7). Interestingly in the present study we found that encoding of both these forms of memory was only impaired with simultaneous inactivation of the NRe inputs to both the $d C A 1$ and $i C A 1$. Importantly this finding demonstrates that the task specificity evident in the CA1 $\rightarrow$ mPFC projection is not determined by information provided by the NRe input but suggests that that the NRe modulates information processing in the HPC and MPFC (either separately or in a coordinated fashion), (8). Additional support for the modulatory role of the NRe is provided by evidence that stimulation of NRe appears to affect activity in inhibitory interneurons in the HPC rather than directly influencing CA1 pyramidal cell firing $(31 ; 32 ; 33$; but see 34$)$. In summary our findings together demonstrate that encoding of spatial and temporal associative information requires the direct $\mathrm{NRe} \rightarrow \mathrm{mPFC}$ pathway and the $\mathrm{NRe} \rightarrow \mathrm{CA} 1 \rightarrow \mathrm{mPFC}$ circuit, and through these pathways the NRe functions to maintain stable memory representations.

Retrieval, but not encoding of both object-in-place and temporal order memory was dependent on the mPFC $\rightarrow$ NRe pathway. In addition, by showing that animals were only amnestic when the light stimulation was applied, we confirmed that the manipulation did 
not erase the memory trace, but rather specifically affected the animals' ability to retrieve information. Thus, these findings provide direct evidence that the accessibility of stored information is mediated through top-down control by the MPFC $(1 ; 15)$. Retrieval was also impaired following inactivation of the $\mathrm{NRe} \rightarrow \mathrm{CA} 1$ pathway, indicating the existence of a $\mathrm{mPFC} \rightarrow \mathrm{NRe} \rightarrow \mathrm{CA} 1$ retrieval circuit. Interestingly, unlike encoding, retrieval of object-inplace memory and temporal order memory was selectively impaired by inactivation of the $\mathrm{NRe} \rightarrow i \mathrm{CA} 1$ and $\mathrm{NRe} \rightarrow d \mathrm{CA} 1$ pathways respectively mapping the division of function among mPFC projecting CA1 neurons (7). That the NRe modulation of CA1 function during retrieval is task specific, suggests that the NRe is required for the activation of the original engram in CA1, a process mediated by the top-down influence of the mPFC.

Memory encoding and retrieval are highly dynamic ongoing processes, thus for successful discrimination between the old and new object-place configurations the animal will be both retrieving old and encoding new information at the same time. Here we used a modified object-in-place task with two test phases, to measure the impact of disruption of either the $\mathrm{mPFC} \rightarrow \mathrm{NRe}$ retrieval or NRe $\rightarrow$ mPFC encoding pathway, on the animal's ability to encode the new or retrieve the old object-place information. We found that blocking encoding had no effect on the animals' ability to simultaneously retrieve information, and vice versa, thus supporting our assertion that encoding and retrieval are mediated concurrently through separate networks. Keeping these processes distinct within tightly controlled networks, may be one mechanism whereby new and old information may be kept separate and interference reduced $(35 ; 36)$.

In summary, input pathways to the MPFC and HPC are necessary for encoding, while mPFC and NRe output pathways are necessary for retrieval. That these processes are maintained 
independently in separate processing pathways may serve to enable the formation of robust associations between an object and its spatio-temporal context, which may be modified and updated in response to new information, whilst maintaining a separation of information processing to reduce interference. These results also demonstrate that object-place or temporal order memory representations are set up in the mPFC using input from the CA1, and maintained over the longer term with input from the NRe. In turn the mPFC facilitates memory retrieval through top-down activation of appropriate representations in the HPC via a mPFC-NRe-CA1 pathway. 


\section{REFRENCES}

1. Preston, A.R. \& Eichenbaum H. Interplay of hippocampus and prefrontal cortex in memory. Current Biology 23:R764-R773 (2013)

2. Place, R., Farovik, A., Brockman, M. \& Eichenbaum H. Bi-directional prefrontal hippocampal interactions support context guided memory. Nat. Neurosci. 19, 992-994 (2016)

3. Tonegawa, S., Pignatelli, M., Roy, D.S. \& Ryan, T.J. Memory engram storage and retrieval. Curr. Opin. Neurobiol. 35, 101-109 (2015a)

4. DeNardo, L.A., Liu, C.D., Allen, W.E., Adams, E.L., Friedmann, D., Fu, L., Guenthner, C.J., Tessier-Lavigne, M. \& Luo, L. Temporal evolution of cortical ensembles promoting remote memory retrieval. Nat. Neurosci., 22, 460-469. (2019)

5. Giannotti, G., Heinsbroek, J.A., Yue, A.J., Deisseroth, K. \& Peters, J. Prefrontal cortex neuronal ensembles encoding fear drive fear expression during long-term memory retrieval. Sci Rep. 24, 10709. (2019)

6. Zhao, M.G., Toyoda, H., Lee, Y.S. et al. Roles of NMDA NR2B subtype receptor in prefrontal long-term potentiation and contextual fear memory. Neuron 47, 859872. (2005)

7. Barker, G.R.I. \& Warburton, E.C. Object-in-place associative recognition memory depends on glutamate receptor neurotransmission within two defined hippocampal-cortical circuits: a critical role for AMPA and NMDA receptors in the hippocampus, perirhinal, and prefrontal cortices. Cereb Cortex 25, 472-481. (2015)

8. Barker, G.R.I., Banks, P.J., Scott, H., Ralph, G.S., Mitrophanous, K.A., Wong, L.F., 
Bashir, Z.I., Uney, J.B. \& Warburton, E.C. Separate elements of episodic memory subserved by distinct hippocampal-prefrontal connections. Nat. Neurosci. 20, 242-250 (2017)

9. Hoover, W.B. \& Vertes, R.P. (2007) Anatomical analysis of afferent projections to the medial prefrontal cortex in the rat. Brain Struct. Funct., 212, 149-179

10. Hoover, W.B. \& Vertes R.P. Collateral projections from nucleus reuniens of thalamus to hippocampus and medial prefrontal cortex in rat: a single and double retrograde fluorescent labeling study. Brain Struct. Funct. 217, 191-209. (2012)

11. Swanson, L.W. A direct projection from Ammon's horn to prefrontal cortex in the rat. Brain Res., 217 pp. 150-154(1981)

12. Thierry, A.M., Gioanni, Y., Dégénétais, E. \& Glowinsky J. Hippocampoprefrontal cortex pathway: anatomical and electrophysiological characteristics. Hippocampus 1, 411-419, (2000)

13. Vertes, R.P. Differential projections of the infralimbic and prelimbic cortex in the rat. Synapse, 51, 32-58. (2004)

14. Varela, C., Kumar, S., Yang, J.Y. \& Wilson, M.A. Anatomical substrates for direct interactions between hippocampus, medial prefrontal cortex, and thalamic nucleus reuniens. Brain Struct. Funct. 219, 911-929. (2014)

15. Barker, G.R.I. \& Warburton, E.C. A critical role for the nucleus reuniens in long-term, but not short-term associative recognition memory formation. J. Neurosci 38, 3208-3217 (2018) 
16. Jayachandran, M., Linley, S., Schlecht, M., Mahler, S.V., Vertes, R.P. \& Allen, T.A. Prefrontal pathways provide top-down control of memory for sequences of events. Cell Reports 28, 640-654 (2019)

17. Semon, R., The mneme. (G. Allen \& Unwin, London, 1921), pp. 304.

18. Tonegawa, S., Liu, X., Ramirez, S. \& Redondo, R.L. Memory engram cells have come of age. Neuron $\mathbf{8 7}, 918-31$ (2015)

19. Ben-Yakov A., Yadin Dudai, Y, \& Mayford, M.R. Memory Retrieval in Mice and Men. Cold Spring Harb Perspect Biol. 7, a021790 (2015)

20. Ennaceur, A. One-trial object recognition in rats and mice: methodological and theoretical issues. Behav. Brain Res. 215, 244-54 (2010)

21. Winters, B.D., Saksida, L.M. \& Bussey, T.J. Object recognition memory: neurobiological mechanisms of encoding, consolidation and retrieval. Neurosci Biobehav Rev 32,1055-70 (2008)

22. Warburton, E.C. \& Brown, M.W. Findings from animals concerning when interactions between perirhinal cortex, hippocampus and medial prefrontal cortex are necessary for recognition memory. Neuropsychologia 48, 2262-2272 (2010)

23. Vertes, R.P., Hoover, W.B., Do Valle, A.C., Sherman, A. \& Rodriguez, J.J. Efferent projections of reuniens and rhomboid nuclei of the thalamus in the rat. $J$. Comp. Neurol. 499, 768-796 (2006)

24. Hyman, J.M., Zilli, E.A., Paley, A.M. \& Hasselmo, M.E. Medial prefrontal cortex cells show dynamic modulation with the hippocampal theta rhythm dependent on behavior. Hippocampus 15, 739-49 (2005) 
25. Sigurdsson, T., Stark, K.L., Karayiorgou, M., Gogos, J.A. \& Gordon, J.A. Impaired hippocampal-prefrontal synchrony in a genetic mouse model of schizophrenia. Nature 464, 763-7 (2010)

26. Zielinski, M.C., Shin, J.D. \& Jadhav, S.P. Coherent coding of spatial position mediated by theta oscillations in the hippocampus and prefrontal cortex. J. Neurosci. 39, 4550-4565 (2019)

27. Siapas, A.G., Lubenov, E.V. \& Wilson, M.A. Prefrontal phase locking to hippocampal theta oscillations. Neuron 46, 141-51 (2005)

28. Anderson, K.L., Rajagovindan, R., Ghacibeh, G.A., Meaor, K.J. \& Ding, M. Theta oscillations mediate interaction between prefrontal cortex and medial temporal lobe in human memory. Cereb Cortex 20, 1604-1612 (2010)

29. Dolleman-van der Weel, M.J., Griffin, A.L., Ito, H.T., Shapiro, M.L., Witter, M.P., Vertes, R.P. \& Allen, T,A.) The nucleus reuniens of the thalamus sits at the nexus of a hippocampus and medial prefrontal cortex circuit enabling memory and behavior. Learn. Mem. 26, 191-205 (2019)

30. Banks, P.J., Warburton. E.C. \& Bashir, Z.I. Plasticity in prefrontal cortex induced by coordinated transmission arising from reuniens/rhomboid nuclei and hippocampus. Cereb. Cortex Comm. 2, tgab029 (2021)

31. Dolleman-Van der Weel, M.J., Lopes da Silva, F.H. \& Witter, M.P. Nucleus reuniens thalami modulates activity in hippocampal field CA1 through excitatory and inhibitory mechanisms. J. Neurosci. 17, 5640-50 (1997) 
32. Eleore, L., López-Ramos, J.C., Guerra-Narbona, R. \& Delgado-García, J.M. Role of reuniens nucleus projections to the medial prefrontal cortex and to the hippocampal pyramidal CA1 area in associative learning. PLoS One 6, e23538 (2011)

33. Cruikshank, S.J., Ahmed, O.J., Stevens, T.R., Patrick, S.L., Gonzalez, A.N., Elmaleh, M. \& Connors, B.W. Thalamic control of layer 1 circuits in prefrontal cortex. J. Neurosci. 32, 17813-23 (2012)

34. Bertram, E.H. \& Zhang, D.X. Thalamic excitation of hippocampal CA1 neurons: a comparison with the effects of CA3 stimulation. Neuroscience 92, 15-26 (1999)

35. Hasselmo, M.E. \& Stern, C.E. Theta rhythm and the encoding and retrieval of space and time. Neuroimage, 85, 656-666 (2014)

36. Aggleton, J.P. \& Nelson, A.J.D. Distributed interactive brain circuits for objectin-place memory: A place for time? Brain Neurosci. Adv. 4:2398212820933471 (2020)

Author Contributions E.C.W, G.R.I.B. contributed to the study design, E.C.W and Z.I.B. obtained the grant funding, G.R.I.B., E.C.W., K.G. and S.T. contributed to the behavioural experiments and data collection, G.R.I.B. and K.G. conducted the surgery. E.C.W. and G.R.I.B. wrote the manuscript. All authors discussed and commented on the manuscript.

\section{Acknowledgements}

We thank Dr Clair Booth for help with the experiments; Dr Paul Banks for comments and discussions on the manuscript. The work was supported by grants to E.C.W. and Z.I.B. from 
the Biotechnology and Biology Sciences Research Council ( BB/L001896/1; BB/L02134X/1) and Wellcome Trust (206401/Z/17/Z).

\section{Author Information}

The authors declare no competing financial interests.

Correspondence and requests for materials should be addressed to ECW (e.c.warburton@bristol.ac.uk)

\section{Methods}

Subjects. All experiments were conducted in naïve adult male Lister Hooded rats (Envigo, UK, weighing 300-350g at the start of experiments). The animals were housed in pairs under a 12-h/12-h light/dark cycle (light phase 20.15-08.15). Behavioural training and testing were conducted during the dark phase of the cycle. Food and water were available ad libitum throughout the experiment. All animal procedures were performed in accordance with the United Kingdom Animals Scientific Procedures Act (1986) and associated guidelines. All efforts were made to minimize any suffering and the number of animals used. Each rat was randomly allocated to an experimental group before surgery.

Stereotaxic surgery for optogenetic or chemogenetic manipulation of specific projections. Each rat was anesthetized with isoflurane (4\% induction, maintenance $2-3 \%$ ) and secured in a stereotaxic frame with the incisor bar set at $3.3 \mathrm{~mm}$ below the interaural line. The viral vectors (AAV5-CamKII-eARCH3.0-EYFP, AAV5-CaMKII-EYFP, AAV5-hSyn-hM4Di-mCherry or AAV5-hSyn-mCherry) were injected bilaterally into the following regions using the stereotaxic coordinates relative to bregma: intermediate CA1 (iCA1) region of hippocampus 
(AP -6.3mm, ML $\pm 5.5, \mathrm{DV}-5.6 \mathrm{~mm})$, medial prefrontal cortex (mPFC) (AP +3.0mm, ML \pm $0.75 \mathrm{~mm}, \mathrm{DV}-4.5 \mathrm{~mm}$ ) or nucleus reuniens of thalamus (NRe) (AP $-2.0 \mathrm{~mm}, \mathrm{ML} \pm 1.4 \mathrm{~mm}, \mathrm{DV}-$ $7.5 \mathrm{~mm}$ at a $10^{\circ}$ angle to vertical in the ML plane), in either $2.0 \mu \mathrm{l}$ (iCA1 and $\mathrm{mPFC}$ ) or $1.0 \mu \mathrm{L}$ (NRe) at a rate of $200 \mathrm{~nL} / \mathrm{min}$. Fibre-optic cannulae (200 $\mu \mathrm{M}$ diameter, 0.22 numerical aperture (NA), Doric Lenses, Quebec, Canada) were implanted bilaterally into either the mPFC (AP $+2.4 \mathrm{~mm}, \mathrm{ML} \pm 0.5 \mathrm{~mm}, \mathrm{DV}-3.2 \mathrm{~mm}$ relative to bregma) or NRe (AP $-1.8 \mathrm{~mm}, \mathrm{ML}$ $\pm 2.0 \mathrm{~mm}$, relative to bregma, DV $-6.4 \mathrm{~mm}$ at $15^{\circ}$ from vertical in $\mathrm{ML}$ plane), alternatively for experiments involving chemogenetic manipulation, the guide cannulae were implanted bilaterally into both the dorsal CA1 (dCA1) region of the hippocampus (AP-3.6mm, ML $\pm 2.5 \mathrm{~mm}$, relative to bregma DV $-3.6 \mathrm{~mm}$ at $10^{\circ}$ from vertical in AP plane) and iCA1 (AP $6.3 \mathrm{~mm}, \mathrm{ML} \pm 5.3 \mathrm{~mm}, \mathrm{DV}-4.3 \mathrm{~mm}$ relative to bregma). Fibre-optic or guide cannulae were anchored to the skull by 4 stainless steel skull screws (Plastics One, Bilaney, UK) and dental acrylic. During surgery, each animal was given fluid replacement therapy $(5 \mathrm{ml}$ saline with $0.9 \%$ glucose s.c.) and analgesia (0.05ml Vetgesic, i.m.).

Stereotaxic surgery for the nucleus reuniens lesion Animals were anaesthetised with isoflurane and placed into a stereotaxic frame with the incisor bar set so as to achieve flat skull. After craniotomy, excitotoxic lesions were made by injection of NMDA $(0.1 \mu \mathrm{l}$ of $0.09 \mathrm{M})$ at the following co-ordinates relative to bregma: AP $-1.72 \mathrm{~mm}$ and $-2.40 \mathrm{~mm}, \mathrm{ML}$ $\pm 0.2 \mathrm{~mm}$ and DV $-7.40 \mathrm{~mm}$, injection occurred gradually over $4 \mathrm{~min}$ and the needle left in situ for further $4 \mathrm{~min}$. for the sham surgery, animals underwent the same surgical procedure as the lesion group with the exception that no excitotoxin was injected ( $n=10$ for all groups). At the completion of experiments animals were perfused and $40 \mu \mathrm{M}$ sections collected as previously described. Sections were mounted and stained using cresyl violet and sections 
were imaged under a light microscope. To assess the extent of the NRe lesion representative sections at the following AP depths relative to bregma were imaged: -1.53 $m m,-2.00 \mathrm{~mm},-2.45 \mathrm{~mm},-2.85 \mathrm{~mm}$ and $-3.25 \mathrm{~mm}$. For each section, the area of each ventral midline structure was measured using imageJ. Lesion size (\% lesion) was calculated for each thalamic nucleus in each animal by comparison to the mean nuclei area in the sham group. The greatest tissue loss was in the NRe (mean \pm SEM, $72 \pm 5.6 \%, \max 93 \%, \min 41 \%$ ), whereas the rhomboid ( $\mathrm{Rh}$ ) and perireuniens nuclei sustained less damage ( $\mathrm{Rh}: 33 \pm 6.3 \%$, $\max 64 \%, \min 4 \%$, perireuniens $28 \pm 7.3 \%, \max 72 \%, \min 0 \%)$.

Optical inhibition. Pathway specific optogenetic inhibition experiments were carried out using $10 \mathrm{~mW}, 515 \mathrm{nM}$ light pulses (10ms duration, $50 \%$ duty cycle), delivered via a $45^{\circ}$ tipped, $200 \mu \mathrm{m}$ diameter, 0.22 NA fibre optic fibre. Light was delivered by diode laser (Omicron LuxX515.100, 515nM, 100mW, Photonlines, UK) connected via a fibre-optic patchcord (200 $\mu \mathrm{M}$ diameter, 0.22NA, Doric lenses, Quebec, Canada) to a 50-50 intensity division fibre-

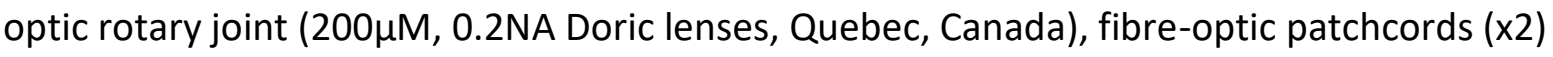
(200 $\mu \mathrm{M}, 0.2 \mathrm{NA})$ connected the splitter to the implanted fibre-optic cannula. TTL pulses to control light delivery were generated using WinLTP v1.1 software (Anderson \& Collingridge 2007) and transmitted to the laser via a BNC-2120 connector (National instruments, Texas, USA). Measurement of light intensity output at the tip of an un-implanted fibre-optic cannula indicated a light power of $10 \mathrm{~mW}$ per channel when the laser was at full power $(100 \mathrm{~mW})$. When animals were to receive laser light delivery they were moved into the experimental room, the dust cap covering the optical cannula was removed and the patch cord was inserted and screwed into place. Light delivery was initiated, and the animal was immediately placed into the arena ( $<5$ s after laser activation), at the completion of the session the animal was promptly removed from the arena and then light delivery was 
terminated ( $<5$ s after removal from arena), the fibre-optic patch-cord was unscrewed and removed and the dust-cap was replaced. For laser off condition the patch-cord was connected to the optical cannula, but the light delivery was not triggered. After each trial, optical connections were lightly cleaned with iso-propyl alcohol.

Chemogenetic inhibition. General infusion procedures were performed as previously described (Barker et al 08). Clozapine-N-oxide (CNO) (Tocris biosciences, Bristol UK) was dissolved in sterile saline and $0.005 \%$ DMSO and infused at $3 \mu \mathrm{M}$ per site at a volume of 0.5 $\mu \mathrm{l}$, over a $2 \mathrm{~min}$ period. The infusions occurred either $15 \mathrm{~min}$ before the sample phase (so as to affect encoding) or $15 \mathrm{~min}$ before the test phase of the task (so as to affect retreival). Vehicle infusion consisted of sterile saline and 0.005\% DMSO.

Histology. Following completion of experiments, each rat was anesthetized with Euthetal (Rhone Merieux) and transcardially perfused with phosphate-buffered saline followed by $4 \%$ paraformaldehyde. After removal, the brain was post-fixed in paraformaldehyde for $2 \mathrm{~h}$ before being transferred to $30 \%$ sucrose in $0.2 \mathrm{M}$ phosphate buffer. Coronal sections ( $40 \mu \mathrm{m}$, sectioned using a cryostat (LEICA CM 3050 S, Leica Microsystems, Milton Keynes, UK ) were mounted onto positively charged slides, left to dry, counterstained with 4,6-diamidino-2phenylindole (DAPI: $1.5 \mu \mathrm{g} / \mathrm{ml}$ ) and coverslipped with Vectashield (Vector laboratories, Burlingame, California). Viral injection sites, axonal fibres in target region and location of implanted of fibre-optic cannulae were imaged using a Leica DM6B microscope with Hamamatsu Orca Flash 4.0 CMOS camera. Fluorescence images of EYFP expression were overlaid onto images of DAPI stain to allow anatomical localisation of viral expression. Injection of AAV5-hysn-HM4Di-mCherry or AAV5-hysn-mCherry resulted in a weak fluorescent signal in axonal projection fibres therefore to image mCherry expression fully in 
these experiments immunofluorescence staining was performed. Free floating coronal sections (40 $\mathrm{mm}$ thick) were washed in 01M PBS wi9th 0.2\% Triton 100 (PBST) and were then blocked for $1 \mathrm{~h}$ (5\% normal goat serum, 2.5\% BSA in PBST. Sections were incubated in primary antibody solution (anti-mCherry 1:1000 Dilution, Abcam) for $24 \mathrm{~h}$ at room temperature. Sections were washed in PBST (four times) and then incubated in secondary antibody either goat anti-rabbit ALEXA Fluor594 (Abcam, Cat No. ab175471) 1 in 250 dilution for $4 \mathrm{~h}$ or biotinylated goat-anti rabbit (Vector, Cat No. BA-1000-1.5) 1 in 200 dilution for $2 \mathrm{~h}$. Sections incubated in ALEXA Fluor594 secondary antibdy were washed in PBST (four times) and then mounted onto polylysine coated slides and cover slipped with vectashield when dry. Sections incubated in biotinylated antibody were washed in PBST (four times) then incubated in avidin-biotin complex ( $A B C$, Vector Laboratories) for $1 \mathrm{~h}$ then washed in PBST (four times) followed by $0.05 \mathrm{M}$ TRIS buffer (two times) before visualisation with DAB (Sigma-Aldrich). Sections were mounted onto polylysine coated slides and when dry were dehydrated and coverslipped with DPX (Thremo-Fisher).

\section{Effect of optical inhibition on immediate early gene expression.}

Expression of the immediate early gene c-fos in MPFC was assessed following deactivation of projections from NRe. Animals were injected with either AAV5-CaMKII-eARCH3.0-EYFP or AAV5-CaMKII-EYFP in NRe and optical cannula were targeted at mPFC. Animals were given a single object-in-place sample phase of 5 min duration with unilateral laser light delivery throughout the session. Ninety minutes after the end of the sample phase, animals were transcardially perfused with $0.1 \mathrm{M}$ PB followed by $4 \%$ PFA and the brains removed. Brains were post-fixed for 2 hours in 4\% PFA and were then transferred to $30 \%$ sucrose solution for $48 \mathrm{~h}$ for cryoprotection. Brains were flash frozen using isopentane and liquid nitrogen and 
were then sectioned at $40 \mu \mathrm{m}$ on a cryostat. Every fifth section from the mPFC was taken for Fos staining. Briefly sections were washed in $3 \% \mathrm{H}_{2} \mathrm{O}_{2}$ followed by PBST $(0.2 \%)$ and were then blocked for $1 \mathrm{~h}$ ( $5 \%$ normal goat serum, $2.5 \%$ BSA in PBST). Sections were then incubated in primary antibody solution (anti c-fos 1:20000 dilution, Abcam Cat No. ab190289) for $48 \mathrm{~h}$ at $4^{\circ} \mathrm{C}$. Sections were washed $4 \mathrm{x}$ with PBST and then incubated in secondary antibody (Biotinylated goat anti-rabbit (Vector Laboratories) 1:200 dilution, washed in PBST then incubated in ABC (Vector Laboratories) for $1 \mathrm{~h}$, washed in PBST followed by 0.1 M TRIS buffer, antibody binding was visualised by reaction with DAB (SigmaAldrich). Sections were mounted on polylysine coated slides allowed to dry and then dehydrated and coverlsipped with DPX (Thermo-Fisher). Images of Fos stained nuclei were taken at x200 magnification using a Leica DM6B microscope with Hamamtsu Orca Flash 4.0 CMOS camera. The two sections closest to the optical cannula tip were imaged in each hemisphere. The number of Fos positive nuclei were counted using image (NIH). Counts were made with the experimenter blind to the experimental condition of each section. Normalised counts were calculated by dividing the number of each Fos positive nuclei in each section by the mean number of positive Fos nuclei in the laser off hemisphere.

Behavioural protocols. Object exploration occurred in a wooden open-topped arena 90 $x 100 \mathrm{~cm}$ with walls $50 \mathrm{~cm}$ high and was video recorded for subsequent analysis. The stimuli presented were objects composed of Duplo blocks (Lego UK Ltd, Slough, UK) that varied in shape, colour and size $(9 \times 8 \times 7 \mathrm{~cm}$ to $25 \times 15 \times 10 \mathrm{~cm})$ and were too heavy for the animal to displace. Objects were cleaned with absolute ethanol between each phase to remove olfactory cues. 
Habituation. For $4 \mathrm{~d}$ before the commencement of behavioural testing the animals were placed in the arena for $5 \mathrm{~min}$.

Object-in-place memory task (Figure 1c). The task comprised a sample and a test phase separated by a 5 min or a $3 \mathrm{~h}$ delay. In the sample phase the rats explored four different objects for $5 \mathrm{~min}$. In the test phase ( 3 min duration), two of the objects, either the two objects on the left or the right (relative to the wall over which the animal entered the arena), switched locations. The time spent exploring the two objects that switched locations was compared to the time spent exploring the two objects that had remained in the same position.

Object-in-place task with two test phases (Figure 3a). The task comprised a sample phase and two test phases, each separated by a $3 \mathrm{~h}$ delay. The sample phase was identical to the standard object-in-place task. In the first test phase, as in the standard object-in-place task, one pair of objects, either on the left or the right of the arena switched locations, the animal was allowed to explore the objects for $5 \mathrm{~min}$. The first test phase was extended to $5 \mathrm{~min}$ duration to give the animals the same amount of time to encode the new object location pair as they had in the sample phase to encode the original object-location associations. In the second test phase ( 3 min duration) the pair of objects which remained stationary in the first test phase now switched locations (novel object-location pair) and the objects which had switched location in the first test phase remained in the same positions as the first test phase (familiar object-location pair).

Object temporal order memory task (Figure 1g). This task involved four sample phases (S1S4) each separated by a $1 \mathrm{~h}$ delay and a test phase which occurred with a $2 \mathrm{~h}$ delay after $\mathrm{S} 4$. In each sample phase the rats explored two copies of an object for $4 \mathrm{~min}$. Different objects 
were presented in each sample phase. In the test phase $(3 \mathrm{~min})$ the rat was presented with objects from S2 and S3 and the time spent exploring each object was recorded.

Object recognition task. To assess recognition memory in the iCA $\rightarrow \mathrm{mPFC}, \mathrm{NRe} \rightarrow \mathrm{mPFC}$ and $\mathrm{mPFC} \rightarrow$ NRe groups a modified object-in-place task was used (Supplementary Data Figure 2e): four different objects were presented in the sample phase (5 min duration), and at test, which occurred following a $3 \mathrm{~h}$ delay, two objects were replaced by novel objects. Thus, at test the animals were presented with two novel objects and two familiar objects. As for the other tasks, the time spent exploring each object was recorded. To assess recognition memory in the $\mathrm{NRe} \rightarrow d \& i \mathrm{CA} 1$ groups a standard object recognition task was used (Supplementary data Figure 5e), in the sample phase the rat was exposed to two identical copies of an object for 4 min or until 40 sec of exploration had been completed. The test phase occurred following a $3 \mathrm{~h}$ delay. In the test phase the rat was placed back in the arena which contained a third copy of the object presented in the sample phase and a novel object. The time spent exploring each object was recorded.

Object location task (Supplementary Data Figure $5 c$ ). In the sample phase the rat was presented with two identical objects for 4 min. The test phase occurred following a delay of $3 \mathrm{~h}$. In the test phase the rat was placed back in the arena, which contained an identical object from the sample phase in the same position as in the sample phase and a fourth identical object in a novel location. The time spent exploring each object was recorded.

Experimental design. In the optogenetic experiments a two-way design with a between subject and within subject factor was used. The between subject factor was virus, (YFP or $A R C H)$, which was assigned randomly at surgery, the within subject factor was laser on or off. Each experiment consisted of two trials separated by a minimum of $6 \mathrm{~d}$. Each animal 
received no laser light (control condition) in one trial and laser light delivery in the other. The choice of laser light status for each experiment was determined randomly. Laser status was counterbalanced across animals within each viral condition so that equal numbers of animals received or did not receive laser stimulation. In the experiment where chemogenetic manipulation was used to deactivate the $\mathrm{NRe} \rightarrow \mathrm{CA} 1$ projection, a four-way within subject design was used. Thus, each experiment consisted of four trials. Each animal received either a control infusion (vehicle into both $d C A 1$ and $i C A 1$ ), CNO infusion into $d C A 1$ only, infusion of CNO into iCA1 only or infusion of CNO into both dCA1 and iCA1. Choice of infusate for each experiment was determined by the experimenter conducting the infusions. Infusates were counterbalanced across animals so that equal numbers of animals received each infusion condition in each run of the task. Within each behavioural experiment the location and/or order of object presentation were also counterbalanced.

Assessment of exploration in behavioural tasks. For all the spontaneous exploration tasks, the proportion of time each animal spent exploring each object was analysed. Exploratory behaviour was strictly defined as the animal directing its nose toward the object at a distance of $<2 \mathrm{~cm}$ and was scored by the experimenter blind to the virus or infusate status of each animal. Object discrimination was determined using a discrimination ratio, calculated as the difference in time spent by each animal exploring the novel compared to the familiar object divided by the total time spent exploring all objects.

Statistical Analysis. The sample size for each experiment was determined by previous studies conducted in both our and other laboratories. Power calculations on previously reported data collected in our laboratory suggested to achieve a power of 0.8 , a group size of eight was required. Larger sample sizes were used to allow for maintenance of power 
should animals be excluded due to cannula misplacement or damage. In the experiments where optogenetics was used to manipulate the pathway initial group sizes were 12 for each virus (i.e. 12 YFP animals and $12 \mathrm{ARCH}$ animals). In the $i \mathrm{CA} 1 \rightarrow \mathrm{mPFC}$ group 4 animals injected with YFP were excluded due to the loss of their implant. In the chemogenetic experiment the starting group size was 16 due to the latin square experimental design used, one animal was excluded for the experiment due to a blocked cannula.

Memory performance between groups was compared using ANOVA analyses using SPSS (IBM). Statistical analyses were designed using an assumption of normal distribution and similar variance but this was not formally tested. Memory performance in the optogenetically manipulated pathways was compared using a two-way mixed design ANOVA with virus as a between subject factor and laser as a within subject factor, post-hoc comparisons analysed the simple main effects. In the object-in-place task with two test phases, memory performance was compared using a three-way mixed-design ANOVA with test phase as a within subject factor in addition to the virus and laser factors used in the two-way analyses, post-hoc comparisons examined simple main effects. Memory performance in the chemo-genetically manipulated pathway was compared using a one-way ANOVA with treatment as a within subject factor, post-hoc tests used a Bonferroni correction. In addition to test whether each group of animals could significantly discriminate between objects or pairs of objects in each task, the discrimination ratios of each condition were compared to zero (chance performance) using a one-sample $t$-test (two-tailed). The significance of the results was accepted at $\mathrm{P}<0.05$.

\section{References}


Barker, G.R.I. \& Warburton, E.C. NMDA receptor plasticity in the perirhinal and prefrontal cortices is crucial for the acquisition of long-term object-in-place associative memory. J. Neurosci 28, 2837-2844 (2008)

Anderson, W.W. \& Collingridge, G.L. Capabilities of the WinLTP data acquisition program extending beyond basic LTP experimental functions. J. Neurosci Methods 162, 346-356 (2007) 

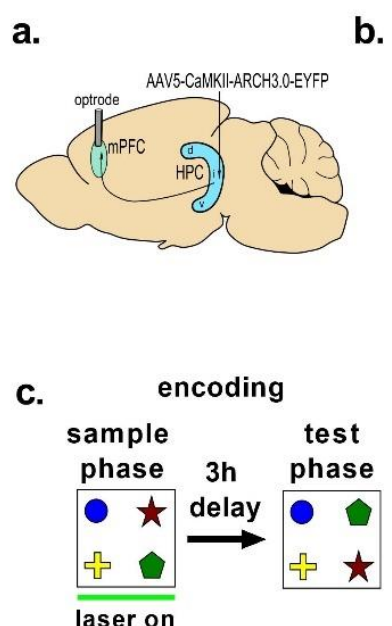

e.

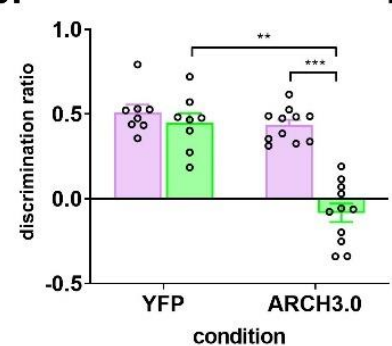

b.

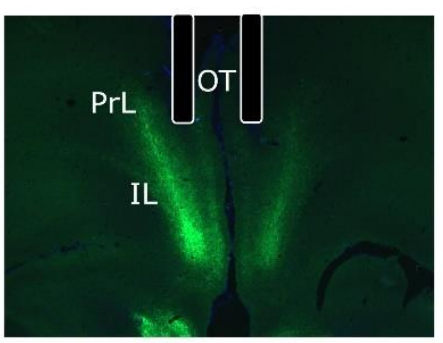

d.

retrieval

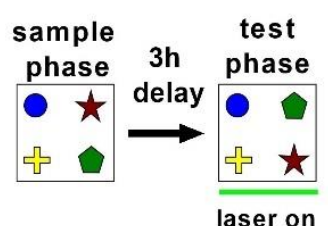

f.

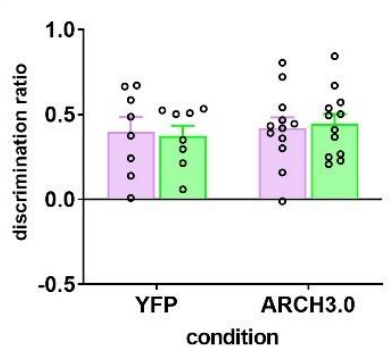

g.

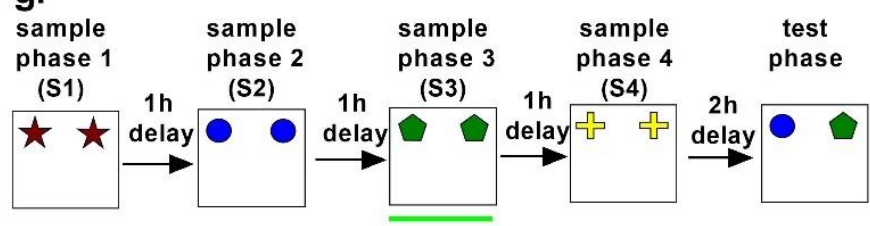

h.

laser on

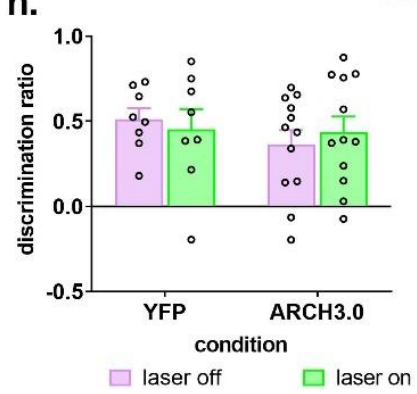

Figure 1: Deactivation of direct projections from intermediate CA1 (iCA1) to medial prefrontal cortex selectively impaired object-in-place memory encoding. (a) Experimental approach to manipulate iCA1 $\rightarrow$ mPFC. (b) Location of optrode and iCA1 axons in mPFC, scale bar: $1000 \mu \mathrm{m}$. (c) Object-in-place task, laser light ( $10 \mathrm{~ms}$ pulse duration, $50 \%$ duty cycle) delivered during the sample phase to test for effects on encoding. (d) Memory impairment when light on during sample (ANOVA laser by virus $F(1,17)=19.07, p=0.001$, YFP $n=8, A R C H n=11$ ). (e) Object-in-place task, laser light delivered during test to test for effects on retrieval. (f) Deactivation of iCA $\rightarrow$ mPFC during test had no effect on performance (ANOVA laser by virus $F(1,18)=0.110, p=0.743$; virus $F(1,18)=0.618, p=0.442$; laser $\mathrm{F}(1,18)=0.001, \mathrm{p}=0.984$, YFP $n=8, A R C H \mathrm{n}=12)$. (g) Object temporal order task with laser delivery during sample phase 3 to affect encoding. (f) Deactivation of iCA $1 \rightarrow m P F C$ during sample phase 3 did not affect performance (ANOVA laser by virus $F(1,18)=0.571, p=0.460$; virus $F(1,18)=0.667, p=0.425$; laser $\mathrm{F}(1,18)=0.008, \mathrm{p}=0.929$ ). All data presented as mean $+\mathrm{SEM}, * * \mathrm{p}<0.01, * * * \mathrm{p}<0.001$. 
a.

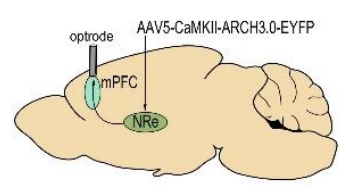

b.

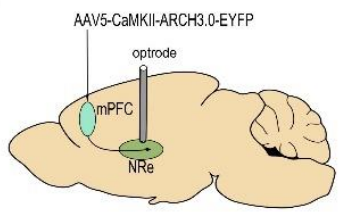

e.
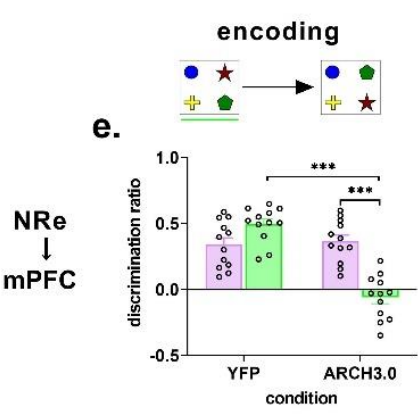

g.

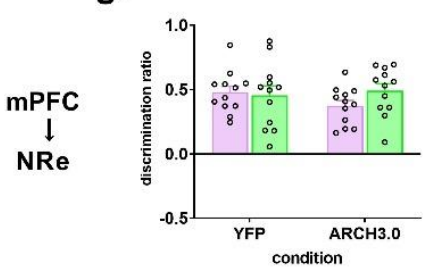

c.

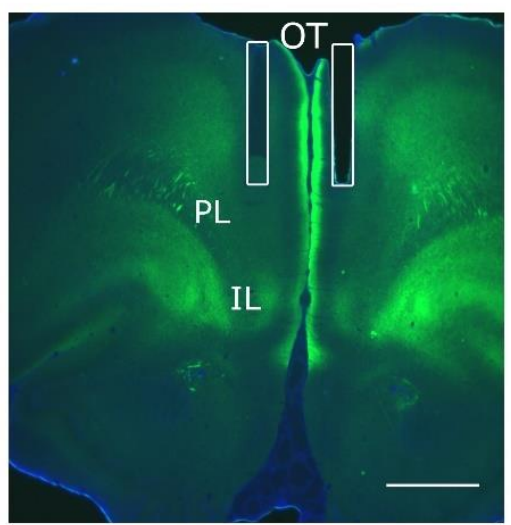

OiP

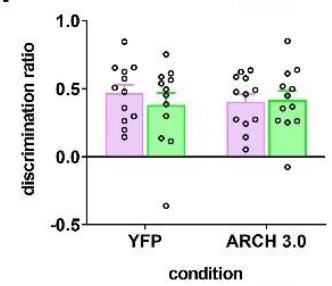

h.

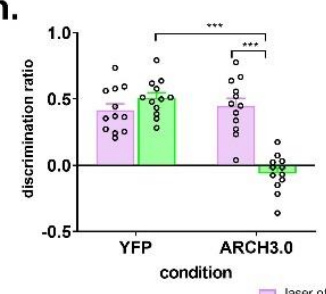

i.

k.

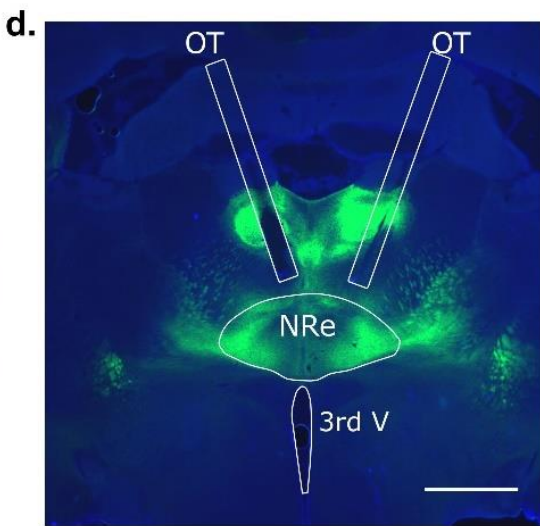

TO
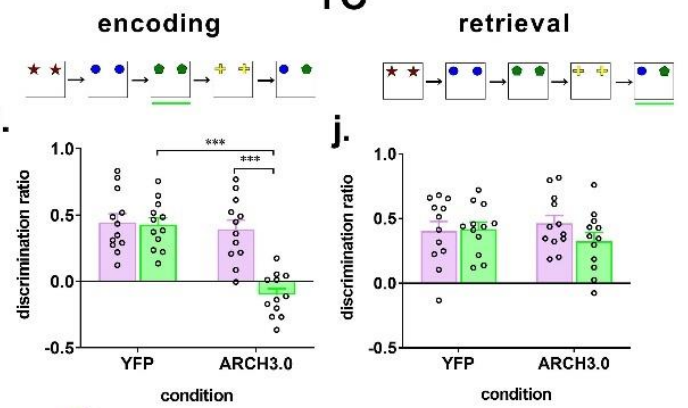

j.
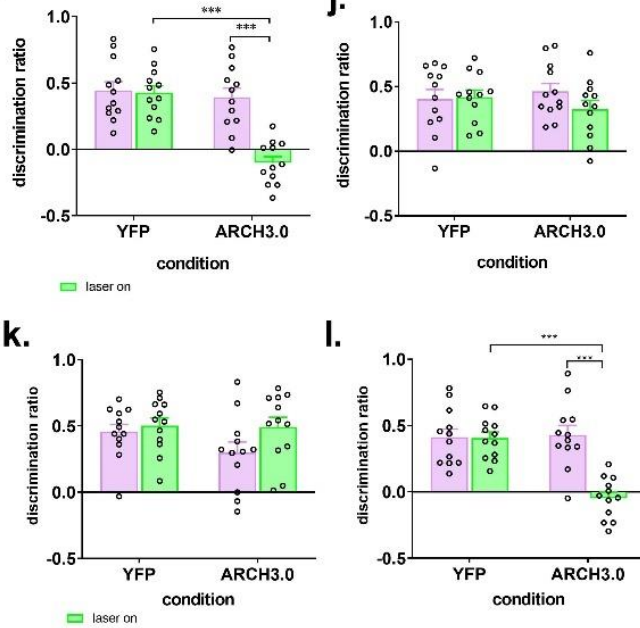

I.

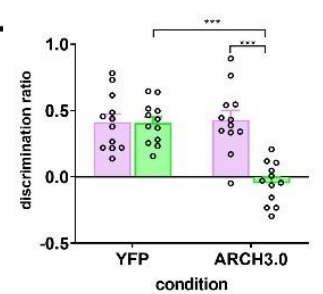

Figure 2: Double dissociation of the role for NRe-mPFC pathways in associative recognition memory encoding and retrieval. (a) Optogenetic manipulation of NRe $\rightarrow$ mPFC. (b) Optogenetic manipulation of mPFC $\rightarrow$ NRe. (c) Optrode location (OT) and NRe axons (shown in green) in mPFC (PL prelimbic; IL infralimbic) scale bar : $1000 \mu \mathrm{M}$. (d) Optrode location (OT) and mPFC axons (shown in green) in NRe (NRe - nucleus reuniens, $3^{\text {rd }} \mathrm{V}$ - third ventricle) scale bar: $1000 \mu \mathrm{M}$. (e) NRe $\rightarrow \mathrm{mPFC}$ inactivation during sample phase significantly impaired performance in the object-in-place task (ANOVA laser by virus $F(1,22)=33.138, p=0.001$ ). (f) Deactivation of $N R e \rightarrow m P F C$ during test had no effect on performance in the object-in-place task (ANOVA laser by virus $F(1,22)=0.501$ ). (g) $\mathrm{mPFC} \rightarrow \mathrm{NRe}$ inactivation during sample phase had no effect (ANOVA laser by virus $F(1,22)=1.510, p=0.232$. (h) Deactivation of $\mathrm{mPFC} \rightarrow$ NRe during test significantly impaired performance in the object-in-place task (ANOVA laser by virus $F(1,22)=47.261, p=0.001$ ). (i) Deactivation of $N R e \rightarrow m P F C$ during sample phase 3 significantly impaired performance in the temporal order task (ANOVA laser by virus $F(1,22)=16.434, p=0.001$ ). (j) Deactivation of $\mathrm{NRe} \rightarrow \mathrm{mPFC}$ during test had no effect on performance in the temporal order task (ANOVA laser by virus $F(1,22)=1.540, p=0.228)$. (k) Deactivation of $\mathrm{mPFC} \rightarrow$ NRe during sample phase 3 had no effect on performance in the temporal order task (ANOVA laser by virus $F(1,22)=1.365$, $\mathrm{p}=0.255$ ). (I) Deactivation of $\mathrm{mPFC} \rightarrow \mathrm{NRe}$ during test significantly impaired performance in the temporal order task (ANOVA laser by virus $F(1,22)=14.668, p=0.001$ ). Data presented as mean + sem, YFP $n=12, A R C H n=12, * * p<0.01, * * * p<0.001$. OiP: object-in-place task, TO: temporal order task. 
a. sample phase

test phase 1

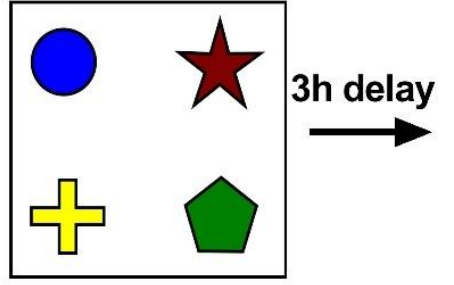

b. $\quad \mathrm{NRe} \rightarrow \mathrm{mPFC}$

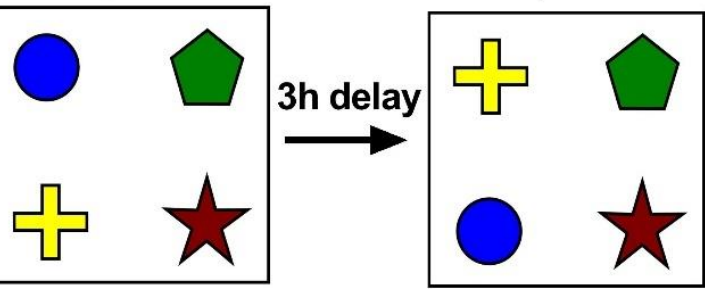

laser on b. $\quad \mathrm{NRe} \rightarrow \mathrm{mPFC}$

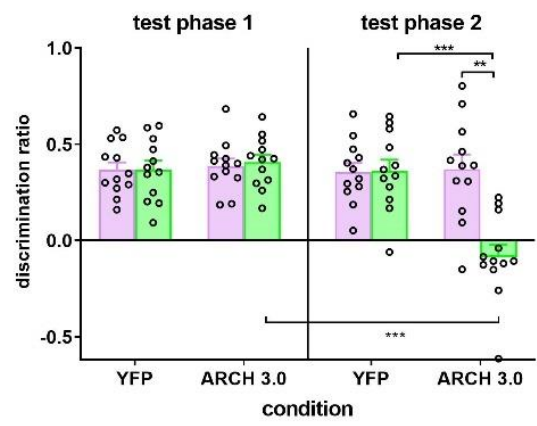

c.

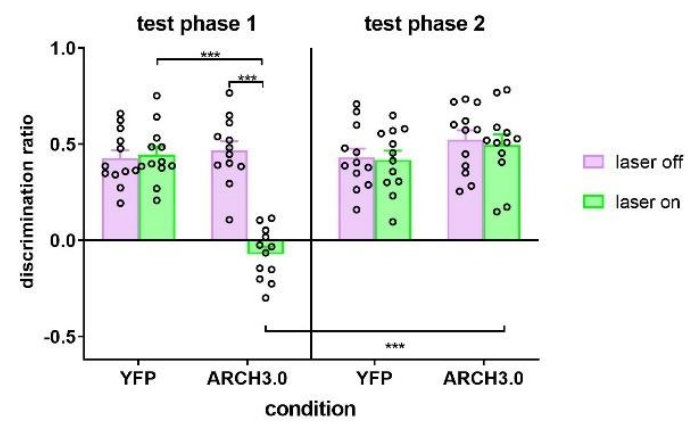

Figure 3: Memory encoding and retrieval can occur independent of one another. (a) Modified objectin-place task with one sample phase and two test phases and laser on during test phase 1. (b) Deactivation of NRe $\rightarrow$ mPFC projections did not affect memory performance during test phase 1 , but significantly impaired performance during test phase 2 (three-way ANOVA virus by laser by test $F(1,22)=13.486, p=0.001$ ). (c) Deactivation of $m P F C \rightarrow N R e$ projections significantly impaired performance during test phase 1 however performance during test phase 2 was not impaired (threeway ANOVA virus by laser by test phase $F(1,22)=15.923, p=0.001$ ). For all experiments, data presented as mean + sem, YFP $n=12$ and $A R C H n=12, * * p<0.01, * * * p<0.001$. 
a.

AAV5-hSyn-hM4Di-mCherry ${ }_{\text {CNO }}$

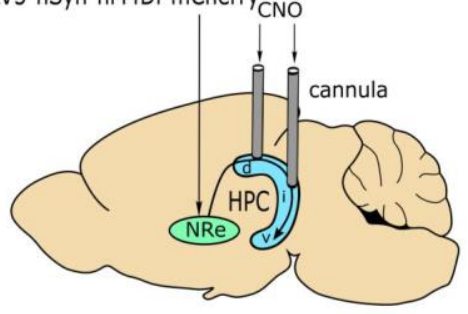

c.

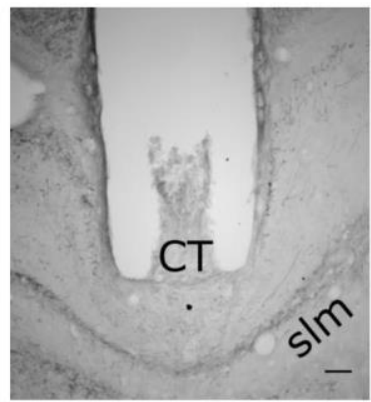

e.

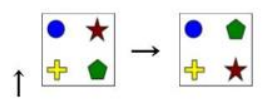

inf.

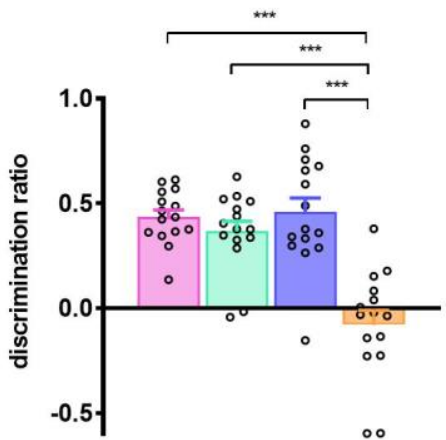

$\square$ Veh d \& iCA1 $\square$ CNO dCA1 $\square$ CNO iCA1 $\square$ CNO d \& iCA1

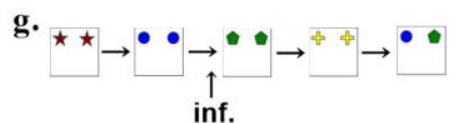

h.

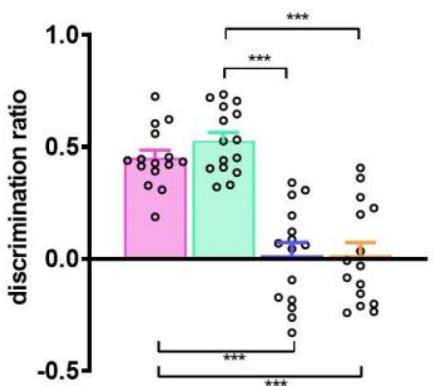

f.

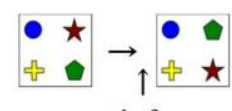
inf.
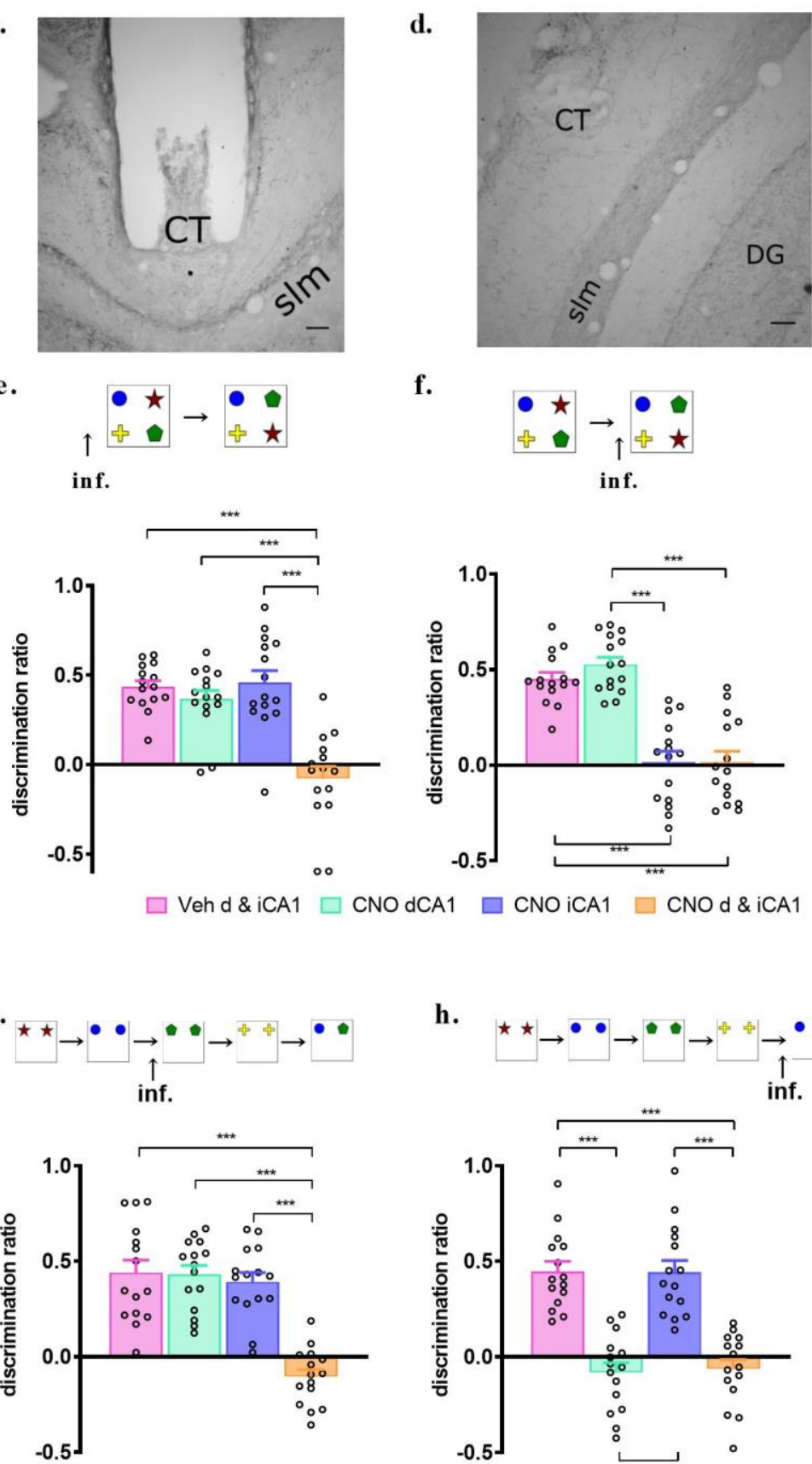

$\square$ Vehd \& iCA1 $\square$ CNO dCA1 $\square$ CNO iCA1 $\square$ CNO d \& iCA 1 
Figure 4: Specific NRe $\rightarrow$ CA1 projections are required for encoding and retrieval of object-in-place and temporal order memory (a) Experimental approach to deactivate projections from NRe to CA1, AAV5-hSyn-hMDi4-mCherry infused into NRe and bilateral cannulae were implanted into dCA1 and iCA1. (b) Location of cannula tract (CT) in $d C A 1$, and fibres expressing hM4Di visible in s/m of the CA1, (DG dentate gyrus) scale bar $1000 \mu \mathrm{M}$. Note the tip of the infusion cannula will extend $1 \mathrm{~mm}$ beyond the end of the guide cannula (c) close up (enlarged from panel b) of cannula and fibres expressing hM4Di in dCA1, scale bar $100 \mu \mathrm{M}$. (d) Location of cannula in iCA1 and fibres expressing hM4Di in slm CA1, scale bar $100 \mu \mathrm{M}$. (e) Infusion of CNO $(0.5 \mu \mathrm{l}$ of $3 \mu \mathrm{M}$ per site) into both dCA1 and iCA1 15 min before the sample phase impaired performance in the object-in-place task (one-way ANOVA $F(3,42)=$ 20.804, $p=0.001$ ). (f) Infusion of CNO 15 min before the test phase significantly impaired performance in the object-in-place task when CNO was infused into either iCA1 or dCA1 \& iCA1, infusion into dCA1 did not significantly alter performance (one-way ANOVA $F(3,42)=38.010, p=0.001$ ). (g) Infusion of CNO 15 min before sample phase 3 significantly impaired performance in the temporal order memory task only when projections to both dCA1 \& iCA1 were deactivated (one-way ANOVA F $(3,42)=26.039$, $p=0.001$ ), deactivation of projections to $d C A 1$ or $i C A 1$ alone did not significantly alter performance. (i) Infusion of CNO $15 \mathrm{~min}$ before the test phase significantly impaired performance in the temporal order memory task when projections to the $d C A 1$ or both $d C A 1$ \& $i C A 1$ were deactivated (one-way ANOVA $F(3,42)=25.918, p=0.001)$, deactivation of projections to $i C A 1$ did not alter performance. For all experiments data presented as mean $+\mathrm{SEM}, \mathrm{n}=15, * * \mathrm{p}<0.01, * * * \mathrm{p}<0.001$. 


\section{Tables}

\begin{tabular}{|c|c|c|c|c|c|c|c|c|}
\hline \multirow{2}{*}{ Task } & \multirow{2}{*}{ Experiment } & \multirow{2}{*}{ Virus } & \multirow{2}{*}{$\begin{array}{l}\text { Laser } \\
\text { status }\end{array}$} & \multicolumn{4}{|c|}{ Exploration in sample phase (s) } & \multirow{2}{*}{$\begin{array}{l}\text { Exploration in } \\
\text { test phase (s) }\end{array}$} \\
\hline & & & & S1 & S2 & S3 & S4 & \\
\hline \multirow{8}{*}{$\begin{array}{l}\text { Object-in- } \\
\text { place }\end{array}$} & \multirow{4}{*}{ Encoding } & \multirow{2}{*}{ YFP } & Off & $80.8 \pm 5.4$ & & & & $37.3 \pm 3.5$ \\
\hline & & & On & $76.4 \pm 8.5$ & & & & $34.7 \pm 3.1$ \\
\hline & & \multirow{2}{*}{$\mathrm{ARCH}$} & Off & $102.8 \pm 8.3$ & & & & $40.1 \pm 3.0$ \\
\hline & & & On & $99.5 \pm 8.2$ & & & & $47.9 \pm 3.6$ \\
\hline & \multirow{4}{*}{ Retrieval } & \multirow{2}{*}{ YFP } & Off & $82.0 \pm 7.7$ & & & & $44.9 \pm 3.5$ \\
\hline & & & On & $75.0 \pm 7.0$ & & & & $40.9 \pm 2.2$ \\
\hline & & \multirow{2}{*}{$\mathrm{ARCH}$} & Off & $105.9 \pm 5.3$ & & & & $43.5 \pm 2.3$ \\
\hline & & & On & $109.8 \pm 5.9$ & & & & $40.6 \pm 2.8$ \\
\hline \multirow{4}{*}{$\begin{array}{l}\text { Temporal } \\
\text { order }\end{array}$} & \multirow{4}{*}{ Encoding } & \multirow{2}{*}{ YFP } & Off & $50.4 \pm 5.2$ & $40.8 \pm 2.5$ & $54.6 \pm 7.6$ & $40.3 \pm 3.0$ & $21.8 \pm 3.9$ \\
\hline & & & On & $47.3 \pm 6.3$ & $46.1 \pm 6.0$ & $49.8 \pm 4.5$ & $50.1 \pm 5.5$ & $20.6 \pm 2.3$ \\
\hline & & \multirow{2}{*}{$\mathrm{ARCH}$} & Off & $67.8 \pm 3.6$ & $57.6 \pm 5.7$ & $57.9 \pm 4.1$ & $52.6 \pm 4.6$ & $29.5 \pm 4.8$ \\
\hline & & & On & $63.5 \pm 7.5$ & $56.7 \pm 5.7$ & $60.7 \pm 5.6$ & $61.0 \pm 7.0$ & $28.0 \pm 3.0$ \\
\hline
\end{tabular}

Table 1: Object exploration in the sample and test phases of the object-in-place and temporal order tasks was not affected following deactivation of iCA1 $\rightarrow$ mPFC projections. Object-in-place encoding sample phase exploration $\mathrm{F}(1,17)=0.02, \mathrm{P}=0.896$; test phase exploration $\mathrm{F}(1,17)=2.73, \mathrm{P}=0.117$; object-in-place retrieval sample phase exploration $F(1,18)=1.88, P=0.187$; test phase exploration $F(1,18)=0.04, P=0.848$; temporal order encoding sample phase exploration $F(3,54)=0.56, P=0.641$; test phase exploration $F(1,18)=0.001, P=0.984$. Data presented as mean \pm sem, $Y F P n=8, A R C H n=12$. 


\begin{tabular}{|c|c|c|c|c|c|c|c|c|c|}
\hline \multirow[b]{2}{*}{ Projection } & \multirow[b]{2}{*}{ Task } & \multirow[b]{2}{*}{ Experiment } & \multirow[b]{2}{*}{ Virus } & \multirow[b]{2}{*}{ Laser } & \multicolumn{4}{|c|}{ Exploration in sample phase (s) } & \multirow{2}{*}{$\begin{array}{l}\text { Exploration } \\
\text { in test } \\
\text { phase }(\mathrm{s})\end{array}$} \\
\hline & & & & & S1 & S2 & S3 & S4 & \\
\hline \multirow{16}{*}{$\mathrm{NRe} \rightarrow \mathrm{mPFC}$} & \multirow{8}{*}{$\begin{array}{l}\text { Object- } \\
\text { in-place }\end{array}$} & \multirow{4}{*}{ Encoding } & \multirow{2}{*}{ YFP } & Off & $95.4 \pm 4.9$ & & & & $37.1 \pm 2.7$ \\
\hline & & & & On & $87.4 \pm 8.7$ & & & & $38.4 \pm 2.8$ \\
\hline & & & \multirow{2}{*}{$\mathrm{ARCH}$} & Off & $95.5 \pm 4.8$ & & & & $36.3 \pm 3.5$ \\
\hline & & & & On & $95.6 \pm 5.5$ & & & & $42.6 \pm 2.1$ \\
\hline & & \multirow{4}{*}{ Retrieval } & \multirow{2}{*}{ YFP } & Off & $82.8 \pm 6.3$ & & & & $42.2 \pm 3.0$ \\
\hline & & & & On & $88.6 \pm 6.1$ & & & & $32.2 \pm 2.4$ \\
\hline & & & \multirow{2}{*}{$\mathrm{ARCH}$} & Off & $89.1 \pm 4.1$ & & & & $36.7 \pm 2.5$ \\
\hline & & & & On & $87.1 \pm 4.6$ & $Z$ & & & $34.6 \pm 2.8$ \\
\hline & \multirow{8}{*}{$\begin{array}{c}\text { Temporal } \\
\text { order }\end{array}$} & \multirow{4}{*}{ Encoding } & \multirow{2}{*}{ YFP } & Off & $61.6 \pm 3.2$ & $46.5 \pm 4.5$ & $37.1 \pm 3.2$ & $51.0 \pm 4.4$ & $24.1 \pm 2.5$ \\
\hline & & & & On & $62.1 \pm 4.4$ & $54.6 \pm 3.6$ & $40.2 \pm 2.7$ & $45.4 \pm 4.4$ & $26.3 \pm 3.0$ \\
\hline & & & \multirow{2}{*}{$\mathrm{ARCH}$} & Off & $57.1 \pm 4.3$ & $47.4 \pm 3.1$ & $39.4 \pm 2.9$ & $51.3 \pm 6.0$ & $31.7 \pm 2.0$ \\
\hline & & & & On & $55.6 \pm 4.2$ & $46.4 \pm 4.6$ & $41.8 \pm 2.8$ & $47.2 \pm 4.6$ & $26.5 \pm 2.5$ \\
\hline & & \multirow{4}{*}{ Retrieval } & \multirow{2}{*}{ YFP } & Off & $62.1 \pm 4.7$ & $61.7 \pm 5.5$ & $55.3 \pm 5.9$ & $48.8 \pm 3.8$ & $31.4 \pm 3.8$ \\
\hline & & & & On & $56.1 \pm 4.1$ & $65.0 \pm 6.5$ & $50.4 \pm 3.5$ & $50.3 \pm 4.0$ & $24.8 \pm 3.2$ \\
\hline & & & \multirow{2}{*}{$\mathrm{ARCH}$} & Off & $59.0 \pm 5.4$ & $59.2 \pm 5.0$ & $56.2 \pm 4.9$ & $48.8 \pm 5.8$ & $26.8 \pm 2.3$ \\
\hline & & & & On & $57.9 \pm 4.4$ & $61.2 \pm 5.4$ & $55.5 \pm 5.9$ & $50.5 \pm 5.4$ & $29.3 \pm 3.5$ \\
\hline \multirow{16}{*}{$\mathrm{mPFC} \rightarrow \mathrm{NRe}$} & \multirow{8}{*}{$\begin{array}{l}\text { Object- } \\
\text { in-place }\end{array}$} & \multirow{4}{*}{ Encoding } & \multirow{2}{*}{ YFP } & Off & $79.0 \pm 3.4$ & & & $Z$ & $37.3 \pm 2.6$ \\
\hline & & & & On & $87.7 \pm 4.8$ & & & & $42.1 \pm 3.0$ \\
\hline & & & \multirow{2}{*}{$\mathrm{ARCH}$} & Off & $82.9 \pm 3.5$ & & & & $37.1 \pm 1.3$ \\
\hline & & & & On & $86.1 \pm 3.3$ & & & & $40.0 \pm 2.5$ \\
\hline & & & & Off & $84.7 \pm 5.0$ & & & & $34.2 \pm 2.1$ \\
\hline & & Retrioval & YFP & On & $92.7 \pm 3.6$ & & & & $37.2 \pm 1.9$ \\
\hline & & Retrieval & & Off & $85.9 \pm 3.8$ & & & & $36.6 \pm 1.7$ \\
\hline & & & $\mathrm{ARCH}$ & On & $85.7 \pm 4.8$ & & & & $37.2 \pm 1.9$ \\
\hline & & & YEP & Off & $69.8 \pm 5.1$ & $62.1 \pm 6.6$ & $44.8 \pm 5.0$ & $54.8 \pm 3.1$ & $34.1 \pm 4.3$ \\
\hline & & & YFP & On & $59.8 \pm 4.2$ & $57.4 \pm 4.8$ & $45.7 \pm 3.1$ & $50.8 \pm 4.2$ & $32.2 \pm 2.5$ \\
\hline & & Encoding & & Off & $62.3 \pm 3.4$ & $49.7 \pm 3.9$ & $44.2 \pm 3.9$ & $41.8 \pm 2.7$ & $28.4 \pm 3.2$ \\
\hline & Temporal & & $\mathrm{ARCH}$ & On & $61.4 \pm 4.0$ & $51.7 \pm 4.2$ & $41.9 \pm 4.0$ & $43.4 \pm 3.4$ & $31.2 \pm 3.1$ \\
\hline & order & & & Off & $69.3 \pm 6.3$ & $53.8 \pm 5.3$ & $62.2 \pm 6.1$ & $56.7 \pm 4.8$ & $33.2 \pm 2.8$ \\
\hline & & & YFP & On & $67.8 \pm 6.1$ & $60.0 \pm 5.8$ & $57.9 \pm 4.2$ & $57.2 \pm 4.5$ & $31.5 \pm 2.4$ \\
\hline & & Retrleval & & Off & $70.9 \pm 5.7$ & $47.8 \pm 4.8$ & $54.0 \pm 3.3$ & $52.1 \pm 7.0$ & $29.0 \pm 3.6$ \\
\hline & & & $\mathrm{ARCH}$ & On & $67.2 \pm 5.5$ & $52.9 \pm 5.4$ & $55 \pm 4.0$ & $57.0 \pm 6.0$ & $31.5 \pm 3.1$ \\
\hline
\end{tabular}

Table 2: Object exploration levels in the sample and test phases of the object-in-place and temporal order task after deactivation of the NRe $\rightarrow \mathrm{mPFC}$ or $\mathrm{mPFC} \rightarrow$ NRe projection. Object-in-place encoding sample phase exploration $\mathrm{NRe} \rightarrow \mathrm{mPFC} F(1,22)=0.44, \mathrm{P}=0.513 ; \mathrm{mPFC} \rightarrow \mathrm{NRe} F(1,22)=0.67, \mathrm{P}=0.420$; test phase exploration $\mathrm{NRe} \rightarrow \mathrm{mPFC} F(1,22)=0.60, \mathrm{P}=0.446 ; \mathrm{mPFC} \rightarrow \mathrm{NRe} F(1,22)=0.12, \mathrm{P}=0.728$; object-in-place retrieval sample phase exploration NRe $\rightarrow \mathrm{mPFC} F(1,22)=0.53, \mathrm{P}=0.473 ; \mathrm{mPFC} \rightarrow \mathrm{NRe}$ $F(1,22)=0.47, P=0.501$; test phase exploration $N R e \rightarrow m P F C F(1,22)=2.87, P=0.104 ; m P F C \rightarrow N R e$ $F(1,22)=0.36, P=0.553$; temporal order encoding sample phase exploration $\operatorname{NRe} \rightarrow m P F C F(3,66)=0.35$, $\mathrm{P}=0.790 ; \mathrm{mPFC} \rightarrow \mathrm{NRe} F(3,66)=0.43, \mathrm{P}=0.734$; test phase exploration $\mathrm{NRe} \rightarrow \mathrm{mPFC} F(1,22)=2.68$, $\mathrm{P}=0.116 ; \mathrm{mPFC} \rightarrow \mathrm{NRe} \mathrm{F}(1,22)=0.42, \mathrm{P}=0.525$; temporal order retrieval test phase exploration $\mathrm{NRe} \rightarrow \operatorname{mPFC} F(3,66)=0.06, \mathrm{P}=0.979 ; \operatorname{mPFC} \rightarrow \mathrm{NRe} F(3,66)=0.13, \mathrm{P}=0.940$; test phase exploration $\mathrm{NRe} \rightarrow \mathrm{mPFC} F(1,22)=1.46, \mathrm{P}=0.240 ; \mathrm{mPFC} \rightarrow \mathrm{NRe} F(1,22)=0.66, \mathrm{P}=0.425$. Data presented as mean \pm sem, $\mathrm{n}=12$ for all conditions. 


\begin{tabular}{|c|c|c|c|c|c|}
\hline \multirow{3}{*}{ Projection } & \multirow{3}{*}{ Virus } & Laser & \multirow{2}{*}{$\begin{array}{c}\text { Exploration in } \\
\text { sample phase }\end{array}$} & \multicolumn{2}{|c|}{ Exploration in test phase (s) } \\
\cline { 4 - 6 } & & & Ts & Test phase 1 & Test phase 2 \\
\hline \multirow{3}{*}{$\mathrm{NRe} \rightarrow \mathrm{mPFC}$} & \multirow{2}{*}{ YFP } & Off & $89.6 \pm 5.8$ & $37.0 \pm 3.3$ & $35.1 \pm 3.8$ \\
\cline { 3 - 6 } & \multirow{2}{*}{ ARCH } & On & $98.8 \pm 4.6$ & $32.9 \pm 2.3$ & $34.0 \pm 2.4$ \\
\cline { 3 - 6 } & & Off & $91.9 \pm 3.8$ & $39.2 \pm 4.3$ & $35.3 \pm 1.6$ \\
\cline { 3 - 6 } & \multirow{2}{*}{ YFP } & on & $85.4 \pm 5.4$ & $34.1 \pm 4.1$ & $38.2 \pm 3.2$ \\
\cline { 3 - 6 } & & Off & $75.2 \pm 4.1$ & $43.1 \pm 4.3$ & $40.4 \pm 3.4$ \\
\cline { 3 - 6 } & \multirow{2}{*}{ ORCH } & On & $68.9 \pm 4.0$ & $46.8 \pm 3.6$ & $39.4 \pm 2.8$ \\
\cline { 3 - 6 } & & Off & $72.3 \pm 5.0$ & $38.7 \pm 3.9$ & $31.0 \pm 3.5$ \\
\cline { 3 - 6 } & & On & $71.8 \pm 4.2$ & $36.3 \pm 2.8$ & $35.7 \pm 4.8$ \\
\hline
\end{tabular}

Table 3: Object exploration in the sample and test phases of the object-in-place task with two test phases, following deactivation of NRe $\rightarrow \mathrm{mPFC}$ or $\mathrm{mPFC} \rightarrow \mathrm{NRe}$ projections. Sample phase exploration $\mathrm{NRe} \rightarrow \mathrm{mPFC} F(1,22)=0.38, \mathrm{P}=0.546 ; \mathrm{mPFC} \rightarrow \mathrm{NRe} \mathrm{F}(1,22)=5.09, \mathrm{P}=0.03$; test phase exploration $\mathrm{NRe} \rightarrow \mathrm{mPFC} F(1,22)=0.51, \mathrm{P}=0.485 ; \mathrm{mPFC} \rightarrow \mathrm{NRe} F(1,22)=1.45, \mathrm{P}=0.241$. Data presented as mean \pm sem, $n=12$ for all conditions.

\begin{tabular}{|c|c|c|c|c|c|c|c|}
\hline \multirow{2}{*}{ Task } & \multirow{2}{*}{ Experiment } & \multirow{2}{*}{ Condition } & \multicolumn{4}{|c|}{ Exploration in sample phase (s) } & \multirow{2}{*}{$\begin{array}{c}\text { Exploration } \\
\text { in test } \\
\text { phase (s) }\end{array}$} \\
\hline & & & S1 & S2 & S3 & S4 & \\
\hline \multirow{8}{*}{$\begin{array}{l}\text { Object- } \\
\text { in-place }\end{array}$} & \multirow{4}{*}{ Encoding } & Vehicle & $94.7 \pm 6.7$ & & & & $36.4 \pm 2.6$ \\
\hline & & CNO- dCA1 & $81.2 \pm 5.1$ & & & & $30.0 \pm 1.8$ \\
\hline & & CNO-iCA1 & $79.0 \pm 4.5$ & & & & $36.7 \pm 2.5$ \\
\hline & & CNO-d\&iCA1 & $81.6 \pm 7.3$ & & & & $37.2 \pm 2.9$ \\
\hline & \multirow{4}{*}{ Retrieval } & Vehicle & $84.5 \pm 5.3$ & & & & $40.9 \pm 2.4$ \\
\hline & & CNO- dCA1 & $82.7 \pm 5.7$ & & & & $40.1 \pm 3.5$ \\
\hline & & CNO-iCA1 & $87.3 \pm 4.0$ & & & & $39.2 \pm 2.9$ \\
\hline & & CNO-d\&iCA1 & $87.3 \pm 7.2$ & 4 & & & $45.3 \pm 4.5$ \\
\hline \multirow{8}{*}{$\begin{array}{c}\text { Temporal } \\
\text { order }\end{array}$} & \multirow{4}{*}{ Encoding } & Vehicle & $67.0 \pm 5.5$ & $57.9 \pm 5.7$ & $52.7 \pm 5.1$ & $47.7 \pm 5.3$ & $31.9 \pm 3.5$ \\
\hline & & CNO- dCA1 & $68.2 \pm 6.5$ & $67.1 \pm 5.7$ & $50.7 \pm 5.6$ & $44.5 \pm 4.9$ & $27.0 \pm 2.9$ \\
\hline & & CNO-iCA1 & $67.9 \pm 5.5$ & $70.3 \pm 8.2$ & $51.9 \pm 5.7$ & $42.6 \pm 4.3$ & $30.9 \pm 4.0$ \\
\hline & & CNO-d\&iCA1 & $68.0 \pm 4.7$ & $58.7 \pm 6.8$ & $60.3 \pm 6.6$ & $53.4 \pm 5.6$ & $35.9 \pm 3.8$ \\
\hline & \multirow{4}{*}{ Retrieval } & Vehicle & $66.5 \pm 4.1$ & $67.6 \pm 6.0$ & $70.1 \pm 5.0$ & $47.0 \pm 4.9$ & $26.4 \pm 2.4$ \\
\hline & & CNO- dCA1 & $64.7 \pm 6.6$ & $60.0 \pm 4.5$ & $62.8 \pm 6.9$ & $41.3 \pm 3.5$ & $30.5 \pm 3.1$ \\
\hline & & CNO-iCA1 & $66.3 \pm 4.9$ & $59.0 \pm 5.9$ & $53.5 \pm 6.1$ & $50.3 \pm 5.1$ & $22.9 \pm 2.0$ \\
\hline & & CNO-d\&iCA1 & $65.1 \pm 6.0$ & $64.8 \pm 6.9$ & $61.5 \pm 4.4$ & $48.9 \pm 3.4$ & $31.9 \pm 3.6$ \\
\hline
\end{tabular}

Table 4: Object exploration levels in the sample and test phases of the object-in-place and temporal order task following deactivation of $\mathrm{NRe} \rightarrow \mathrm{CA} 1$ projections. Object-in-place encoding sample phase exploration $\mathrm{F}(3,42)=3.39, \mathrm{P}=0.03$ (post-hoc veh-dCA1 $\mathrm{P}=0.04$, no other significant differences); test phase exploration $F(3,42)=3.67, P=0.02$ (post-hoc no significant effects); object-in-place retrieval sample phase exploration $F(3,42)=0.38, P=0.768$; test phase exploration $F(3,42)=0.68, P=0.569$; temporal order encoding sample phase exploration $F(9,126)=1.41, P=0.339$; test phase exploration $F(3,42)=0.99, P=0.407$; temporal order retrieval sample phase exploration $F(9,126)=0.87, P=0.548$; test phase exploration $F(3,42)=5.30, P=0.003$ ( $d C A 1$ viCA1 $P=0.009$, iCA1 v diCA1 $P=0.016$ ). Data presented as mean \pm sem, $\mathrm{n}=15$ for all conditions. 


\section{Additional Information}

\section{Supplementary Tables}

\begin{tabular}{|c|c|c|c|c|c|c|c|}
\hline \multirow[b]{2}{*}{ Task } & \multirow[b]{2}{*}{ Delay } & \multirow[b]{2}{*}{ Condition } & \multicolumn{4}{|c|}{ Exploration in sample phase (s) } & \multirow{2}{*}{$\begin{array}{c}\text { Exploration } \\
\text { in test } \\
\text { phase (s) }\end{array}$} \\
\hline & & & S1 & S2 & S3 & S4 & \\
\hline \multirow{4}{*}{$\begin{array}{c}\text { Temporal } \\
\text { order }\end{array}$} & \multirow{2}{*}{$5 \min$} & Sham & $58.8 \pm 3.3$ & $60.2 \pm 5.3$ & $57.4 \pm 6.8$ & $47.3 \pm 6.0$ & $38.8 \pm 6.0$ \\
\hline & & NRe lesion & $61.8 \pm 3.6$ & $70.7 \pm 8.7$ & $59.7 \pm 6.2$ & $40.6 \pm 4.4$ & $40.3 \pm 4.8$ \\
\hline & \multirow{2}{*}{$1 \mathrm{~h}$} & Sham & $54.1 \pm 6.6$ & $41.0 \pm 4.2$ & $42.4 \pm 2.6$ & $33.8 \pm 4.0$ & $19.4 \pm 2.6$ \\
\hline & & NRe lesion & $71.8 \pm 8.0$ & $49.1 \pm 3.2$ & $49.3 \pm 5.8$ & $50.9 \pm 5.8$ & $28.5 \pm 1.6$ \\
\hline
\end{tabular}

Supplementary Table 1: Object exploration levels in the temporal order memory task following lesion of the nucleus reuniens. Temporal order memory sample phase exploration 5 min delay $F$ $(3,48)=1.05, \mathrm{P}=0.379 ; 1 \mathrm{~h}$ delay $\mathrm{F}(3,45)=0.66, \mathrm{P}=0.584$; test phase exploration $5 \mathrm{~min}$ delay $F(1,16)=0.03, P=0.862 ; 1$ delay $F(1,15)=8.38, P=0.011$..Data expressed as mean \pm sem, $n=9$ for both conditions.

\begin{tabular}{|c|c|c|c|c|c|}
\hline Projection & Experiment & Virus & $\begin{array}{l}\text { Laser/ } \\
\text { infusate }\end{array}$ & $\begin{array}{l}\text { Exploration in } \\
\text { sample phase } \\
\text { (s) }\end{array}$ & $\begin{array}{l}\text { Exploration in } \\
\text { test phase (s) }\end{array}$ \\
\hline \multirow{8}{*}{$\mathrm{iCA} 1 \rightarrow \mathrm{mPFC}$} & \multirow{4}{*}{ encoding } & \multirow{2}{*}{ YFP } & Off & $104.0 \pm 5.1$ & $43.5 \pm 3.6$ \\
\hline & & & On & $101.9 \pm 7.7$ & $44.4 \pm 4.7$ \\
\hline & & \multirow{2}{*}{$\mathrm{ARCH}$} & Off & $105.8 \pm 6.4$ & $45.6 \pm 3.7$ \\
\hline & & & On & $102.4 \pm 5.0$ & $35.9 \pm 3.3$ \\
\hline & \multirow{4}{*}{ retrieval } & \multirow{2}{*}{ YFP } & Off & $85.9 \pm 7.4$ & $34.3 \pm 4.1$ \\
\hline & & & On & $88.3 \pm 6.3$ & $36.6 \pm 1.6$ \\
\hline & & \multirow{2}{*}{$\mathrm{ARCH}$} & Off & $96.2 \pm 5.4$ & $36.4 \pm 3.6$ \\
\hline & & & On & $108.8 \pm 6.7$ & $37.1 \pm 3.9$ \\
\hline \multirow{4}{*}{$\mathrm{NRe} \rightarrow \mathrm{mPFC}$} & \multirow{4}{*}{ encoding } & \multirow{2}{*}{ YFP } & Off & $73.7 \pm 3.5$ & $29.8 \pm 3.3$ \\
\hline & & & On & $76.1 \pm 5.4$ & $27.9 \pm 3.3$ \\
\hline & & \multirow{2}{*}{$\mathrm{ARCH}$} & Off & $73.5 \pm 7.0$ & $30.3 \pm 3.6$ \\
\hline & & & On & $88.6 \pm 8.8$ & $27.6 \pm 2.7$ \\
\hline \multirow{4}{*}{$\mathrm{mPFC} \rightarrow \mathrm{NRe}$} & \multirow{4}{*}{ retrieval } & \multirow{2}{*}{ YFP } & Off & $90.1 \pm 7.6$ & $36.3 \pm 2.3$ \\
\hline & & & On & $86.8 \pm 7.9$ & $37.9 \pm 3.2$ \\
\hline & & \multirow{2}{*}{$\mathrm{ARCH}$} & Off & $91.8 \pm 6.5$ & $30.1 \pm 3.4$ \\
\hline & & & On & $96.6 \pm 6.8$ & $39.0 \pm 5.4$ \\
\hline \multirow{2}{*}{$\mathrm{NRe} \rightarrow \mathrm{CA} 1$} & \multirow{2}{*}{ encoding } & \multirow{2}{*}{ hM4di } & Vehicle & $83.9 \pm 4.7$ & $31.8 \pm 4.2$ \\
\hline & & & CNO-d\&iCA1 & $83.2 \pm 6.7$ & $30.8 \pm 3.0$ \\
\hline
\end{tabular}

Supplementary Table 2: Object exploration in the sample and test phases of the object-in-place task with a 5 min delay following deactivation of either iCA $\rightarrow$ mPFC, NRe $\rightarrow$ mPFC, $m P F C \rightarrow$ NRe or $\mathrm{NRe} \rightarrow \mathrm{CA} 1$ projections during either memory encoding or retrieval. iCA1 $\rightarrow \mathrm{mPFC}$ sample phase exploration, encoding $F(1,19)=0.01, P=0.923$; retrieval $F(1,18)=1.17, P=0.294$; test phase exploration encoding $F(1,19)=3.12, P=0.094$; retrieval $F(1,18)=0.05, P=0.822$. NRe $\rightarrow m P F C$ sample phase exploration $\mathrm{F}(1,21)=1.25, \mathrm{P}=0.276$; test phase exploration $\mathrm{F}(1,21)=0.02, \mathrm{P}=0.886$. mPFC $\rightarrow \mathrm{NRe}$ sample phase exploration $F(1,22)=0.41, P=0.528$; test phase exploration $F(1,22)=0.91, P=0.351$. 
$\mathrm{NRe} \rightarrow \mathrm{CA} 1$ sample phase exploration $\mathrm{F}(1,14)=0.01, \mathrm{P}=0.923$; test phase exploration $\mathrm{F}(1,14)=0.04$, $\mathrm{P}=0.841$. Data expressed as mean \pm sem.

\begin{tabular}{|c|c|c|c|c|c|c|}
\hline Projection & Experiment & Virus & $\begin{array}{l}\text { Laser/ } \\
\text { infusate }\end{array}$ & \multicolumn{2}{|c|}{$\begin{array}{c}\text { Exploration in sample } \\
\text { phase (s) }\end{array}$} & $\begin{array}{l}\text { Exploration } \\
\text { in test phase } \\
\text { (s) }\end{array}$ \\
\hline \multirow{4}{*}{$\mathrm{iCA} 1 \rightarrow \mathrm{mPFC}$} & \multirow{4}{*}{$\begin{array}{l}\text { object } \\
\text { recognition } \\
\text { encoding }\end{array}$} & \multirow{2}{*}{ YFP } & off & \multicolumn{2}{|c|}{$73.3 \pm 5.3$} & $44.7 \pm 3.9$ \\
\hline & & & on & \multicolumn{2}{|c|}{$74.0 \pm 7.5$} & $46.2 \pm 2.7$ \\
\hline & & \multirow{2}{*}{$\mathrm{ARCH}$} & off & \multicolumn{2}{|c|}{$96.8 \pm 6.2$} & $56.2 \pm 4.9$ \\
\hline & & & on & \multicolumn{2}{|c|}{$102.2 \pm 5.4$} & $58.2 \pm 3.8$ \\
\hline \multirow{4}{*}{$\mathrm{NRe} \rightarrow \mathrm{mPFC}$} & \multirow{4}{*}{$\begin{array}{l}\text { object } \\
\text { recognition } \\
\text { encoding }\end{array}$} & \multirow{2}{*}{ YFP } & off & \multicolumn{2}{|c|}{$79.6 \pm 3.9$} & $46.4 \pm 4.1$ \\
\hline & & & on & \multicolumn{2}{|c|}{$80.3 \pm 4.2$} & $46.8 \pm 4.0$ \\
\hline & & \multirow{2}{*}{$\mathrm{ARCH}$} & off & \multicolumn{2}{|c|}{$92.5 \pm 5.4$} & $44.3 \pm 2.8$ \\
\hline & & & on & \multicolumn{2}{|c|}{$84.9 \pm 4.1$} & $41.8 \pm 3.3$ \\
\hline \multirow{4}{*}{$\mathrm{mPFC} \rightarrow \mathrm{NRe}$} & \multirow{4}{*}{$\begin{array}{l}\text { object } \\
\text { recognition } \\
\text { retrieval }\end{array}$} & \multirow{2}{*}{ YFP } & off & \multicolumn{2}{|c|}{$101.6 \pm 6.1$} & $55.2 \pm 4.2$ \\
\hline & & & on & \multicolumn{2}{|c|}{$95.2 \pm 6.1$} & $51.6 \pm 2.3$ \\
\hline & & \multirow{2}{*}{$\mathrm{ARCH}$} & off & \multicolumn{2}{|c|}{$99.6 \pm 7.5$} & $46.3 \pm 2.5$ \\
\hline & & & on & \multicolumn{2}{|c|}{$96.6 \pm 5.2$} & $50.1 \pm 3.5$ \\
\hline \multirow{4}{*}{$\mathrm{NRe} \rightarrow \mathrm{CA} 1$} & \multirow{2}{*}{$\begin{array}{c}\text { object } \\
\text { recognition } \\
\text { encoding }\end{array}$} & \multirow{4}{*}{ hM4Di } & vehicle & $147.1 \pm 17.0$ & $37.9 \pm 1.4$ & $36.8 \pm 4.4$ \\
\hline & & & $\begin{array}{c}\text { CNO- } \\
d \& i C A 1\end{array}$ & $141.1 \pm 19.5$ & $38.3 \pm 0.8$ & $36.0 \pm 3.7$ \\
\hline & object & & vehicle & \multicolumn{2}{|c|}{$68.6 \pm 7.3$} & $32.2 \pm 3.7$ \\
\hline & $\begin{array}{l}\text { location } \\
\text { encoding }\end{array}$ & & $\begin{array}{c}\text { CNO- } \\
d \& i C A 1\end{array}$ & \multicolumn{2}{|c|}{$62.1 \pm 6.5$} & $35.8 \pm 4.8$ \\
\hline
\end{tabular}

Supplementary Table 3 Object exploration in the sample and test phases of the object recognition and object location task following deactivation of either iCA1 $\rightarrow \mathrm{mPFC}, \mathrm{NRe} \rightarrow \mathrm{mPFC}, \mathrm{mPFC} \rightarrow \mathrm{NRe}$ or $\mathrm{NRe} \rightarrow \mathrm{CA} 1$ projections. iCA1 $\rightarrow \mathrm{mPFC}$ sample phase exploration $\mathrm{F}(1,18)=0.32, \mathrm{P}=0.577$; test phase exploration $F(1,18)=0.01, P=0.948$, YFP $n=8, A R C H n=12$. NRe $\rightarrow$ mPFC sample phase exploration $F(1,22)=0.77, P=0.39$; test phase exploration $F(1,22)=0.16, P=0.697$, YFP $n=12, A R C H n=12$. $m P F C \rightarrow$ NRe sample phase exploration $F(1,22)=0.13, P=0.72$; test phase exploration $F(1,22)=1.44$, $P=0.243, Y F P n=12, A R C H n=12$. NRe $\rightarrow$ CA1 sample phase exploration, object recognition sample time $F(1,14)=0.10, P=0.762$; exploration in sample phase $F(1,14)=0.20, P=0.661$; object location $F(1,14)=0.53, P=0.477$; test phase exploration, object recognition $F(1,14)=0.03, P=0.873$; object location $F(1,14)=0.437, P=0.52$, vehicle and $C N O n=15$. 


\begin{tabular}{|c|c|c|c|c|c|}
\hline \multirow[b]{2}{*}{ Virus } & \multirow[b]{2}{*}{ Laser } & \multirow{2}{*}{$\begin{array}{l}\text { Exploration in } \\
\text { sample phase } \\
\text { (s). }\end{array}$} & \multicolumn{3}{|c|}{ Exploration in test phase (s). } \\
\hline & & & $\begin{array}{c}\text { First } 3 \\
\text { minutes }\end{array}$ & $\begin{array}{c}\text { Second } 3 \\
\text { minutes }\end{array}$ & total \\
\hline \multirow{2}{*}{ YFP } & off & $91.3 \pm 7.0$ & $36.8 \pm 2.7$ & $25.9 \pm 2.8$ & $62.7 \pm 4.0$ \\
\hline & on & $92.0 \pm 5.4$ & $43.1 \pm 3.5$ & $23.9 \pm 1.9$ & $67.0 \pm 4.2$ \\
\hline \multirow{2}{*}{$\mathrm{ARCH}$} & off & $92.9 \pm 5.9$ & $39.0 \pm 3.7$ & $16.1 \pm 1.8$ & $55.1 \pm 4.4$ \\
\hline & on & $88.9 \pm 6.2$ & $38.2 \pm 3.8$ & $20.9 \pm 2.4$ & $59.2 \pm 3.7$ \\
\hline
\end{tabular}

Supplementary Table 4: Object exploration levels in the sample and test phase of the object-in-place task where $\mathrm{mPFC} \rightarrow \mathrm{NRe}$ projections were deactivated for the first three minutes of the test phase. Exploration in sample phase $\mathrm{F}(1,22)=0.37, \mathrm{P}=0.552$; exploration in test phase, first three minutes $F(1,22)=1.54, P=0.228$; second three minutes of test phase $F(1,22)=3.77, P=0.065$; total exploration in test 


\section{Supplementary Figures}

a.

\section{Laser}

off

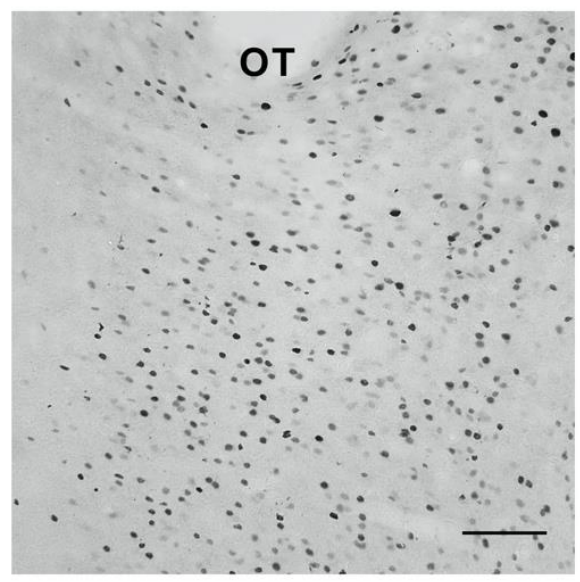

YFP

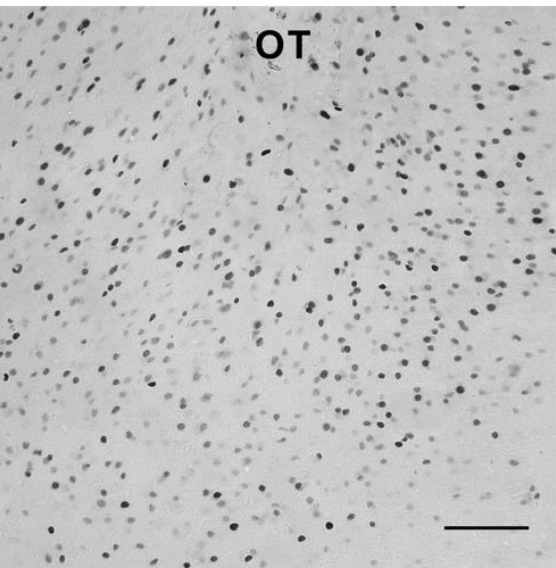

on
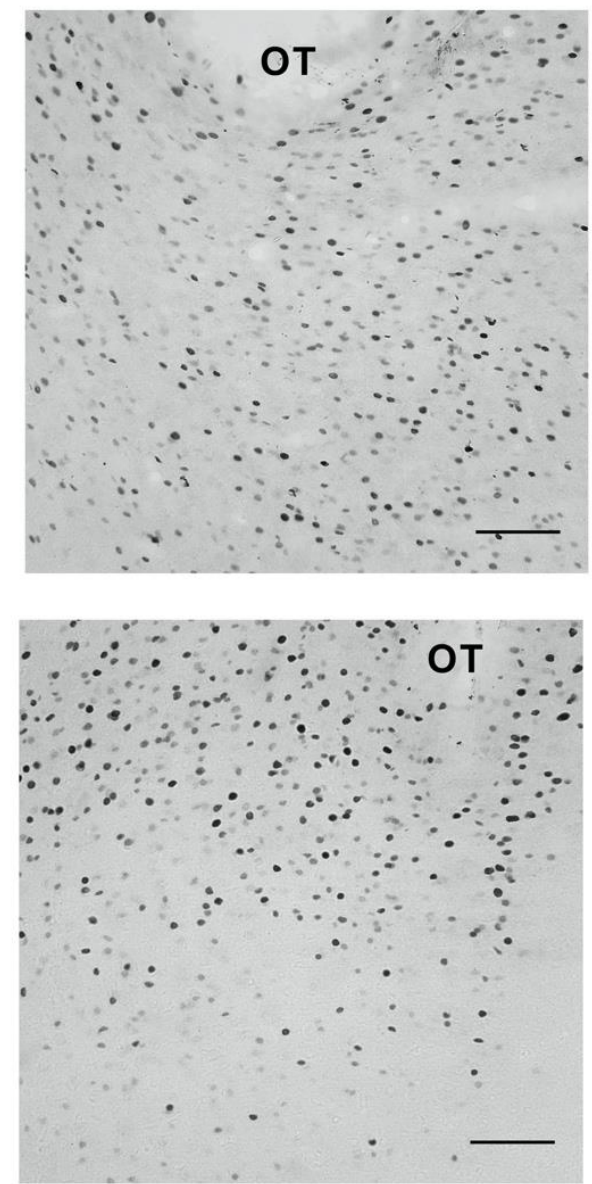

c.

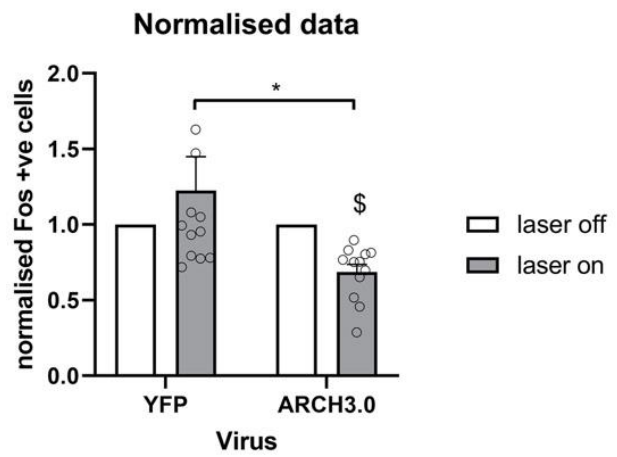

Supplementary Figure 1: Deactivation of the projection from NRe to MPFC significantly reduces Fos expression in mPFC. (a) Representative photomicropraph of coronal sections showing Fospositive cells in MPFC following deactivation of NRe MPFC projections. Sections show the region of prelimbic cortex, ventral to the optrode tip (OT). (b) Fos raw counts were significantly reduced in animals transfected with ARCH in the brain hemisphere in which the laser light was delivered (twoway ANOVA laser by virus interaction $F(1,22)=9.674, p=0.005$, laser $F(1,22)=4.705, p=0.041$, virus 
$F(1,22)=2.006, p=0.171)$ compared to the hemisphere in which laser light was not delivered, in animals transfected with YFP laser light delivery did not significantly alter Fos expression levels. (c) Normalised Fos counts show significantly reduced Fos expression following transfection with ARCH and laser light delivery (one-way ANOVA $F(1,22)=5.526, p=0.028$; one sample t-test YFP $t(11)=1.01$, $\mathrm{p}=0.335, \mathrm{ARCH} t(11)=-6.066, \mathrm{p}=0.0001)$. Data expressed as mean +sem (bar and error bar) and individual values (open circles), YFP and ARCH N=12, OT: optrode tip, scale bar $100 \mu \mathrm{M} .{ }^{*} \mathrm{p}<0.05,{ }^{*}$ $p<0.01, * * * p<0.001, \$$ significance in the one-sample t-test against 1 
a.

sample phase

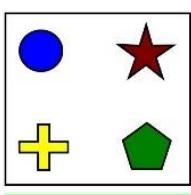

laser on

C.
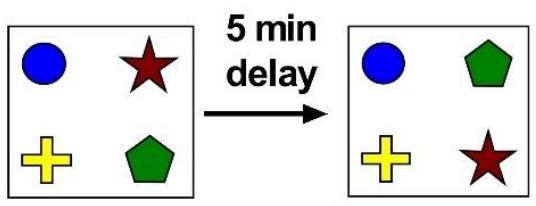

laser on b.

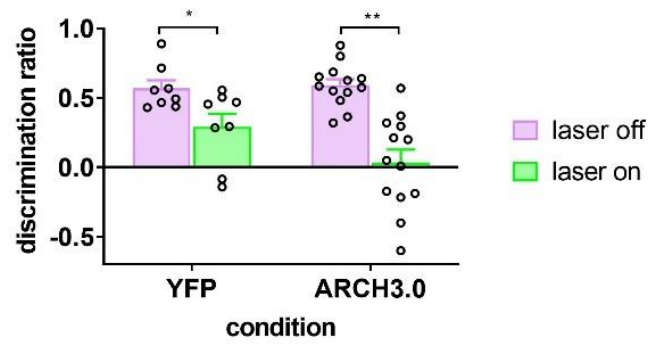

d.

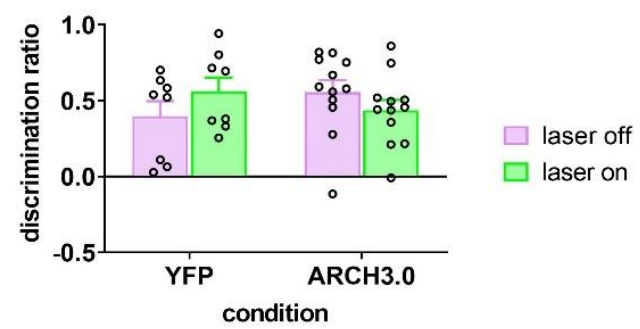

f.

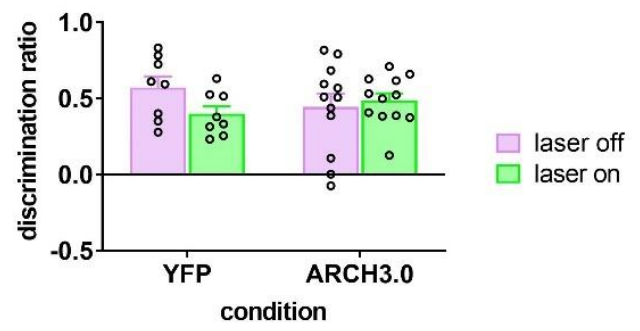

Supplementary Figure 2: Projections from iCA1 to mPFC are required for the encoding but not retrieval of associative recognition memory or the encoding of non-associative recognition memory. (a \& c) Outline of object-in-place memory task with a 5-min delay between sample and test phase, to test memory encoding (a) laser light was delivered during the sample phase, to test memory retrieval laser light was delivered during the test phase (c). (b \& d) Deactivation of iCA1 $\rightarrow$ mPFC projections significantly impaired memory encoding but not retrieval, (c) performance in the object-in-place task was significantly impaired when iCA $1 \rightarrow \mathrm{mPFC}$ projections were deactivated during memory encoding (two-way ANOVA laser $F(1,19)=29.060, p=0.001$; virus by laser $F(1,19)=3.259, p=0.087$; virus $F(1,19)=$ $2.167 p=0.157$, YFP $n=8, A R C H n=13$ ), in contrast (d) performance was not significantly altered when projections were activated during memory retrieval (Two-way ANOVA virus by laser $F(1,18)=2.664$, $p=0.120$; virus $F(1,18)=0.047, p=0.830$; laser $F(1,18)=0.063, p=0.805$, YFP $n=8, A R C H n=12$ ). (e) Outline of the object recognition memory task based on the object-in-place task, laser light was delivered during the sample phase to test the effect on memory encoding. (f) Deactivation of iCA $1 \rightarrow m P F C$ projections during memory encoding did not alter object recognition memory performance (two-way ANOVA virus by laser $F(1,18)=3.294, p=0.086$; virus $F(1,18)=0.058, p=0.812$; laser $F(1,18)=1.207$, $\mathrm{p}=0.286, \mathrm{YFP} n=8, A R C H \mathrm{n}=12$ ). Data presented as mean + sem (bar and error bar) and individual values (open circles). ${ }^{*} \mathrm{p}<0.05,{ }^{* *} \mathrm{p}<0.01,{ }^{* * *} \mathrm{p}<0.001$ 


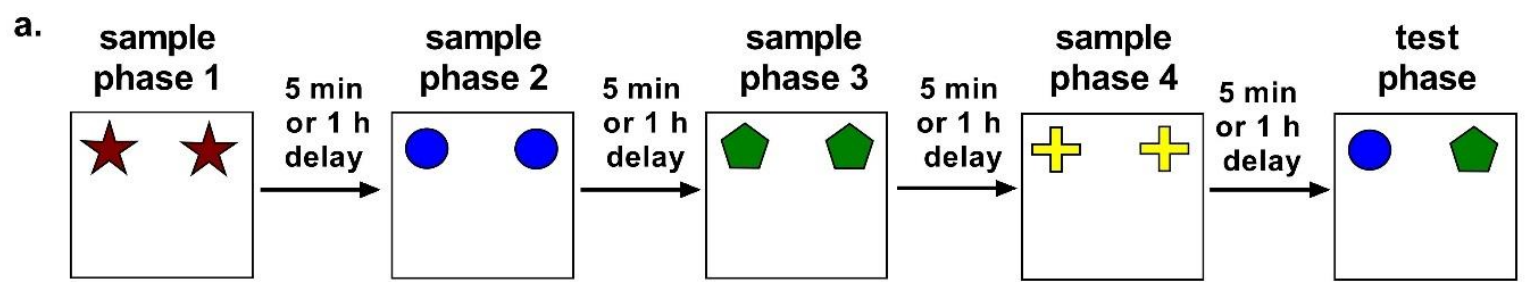

b.

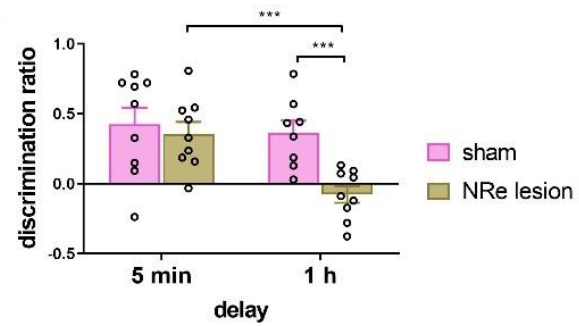

c.

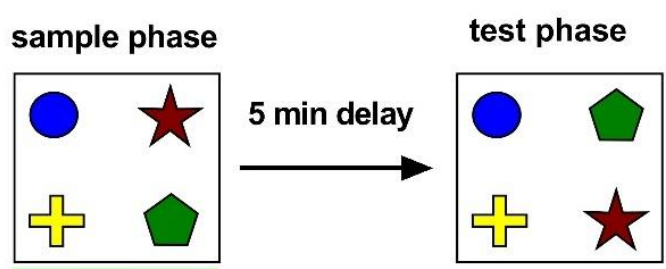

laser on

e.
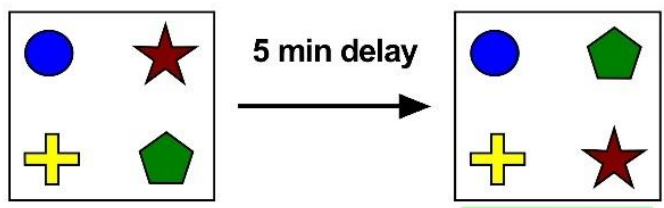

laser on

g.

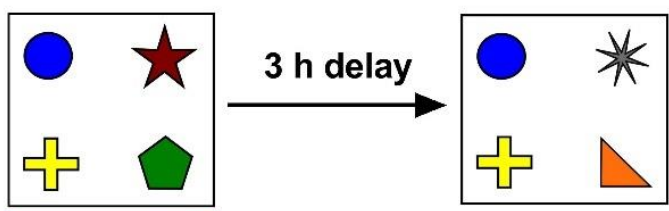

i.

laser on

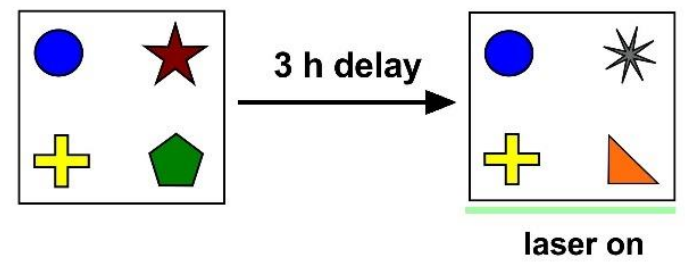

d.

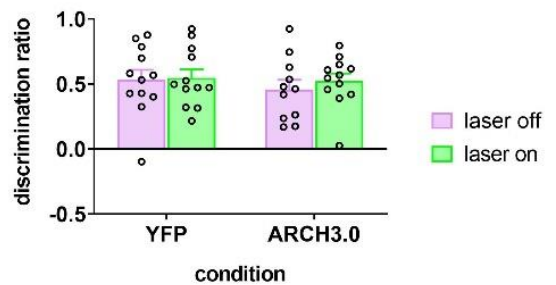

f.

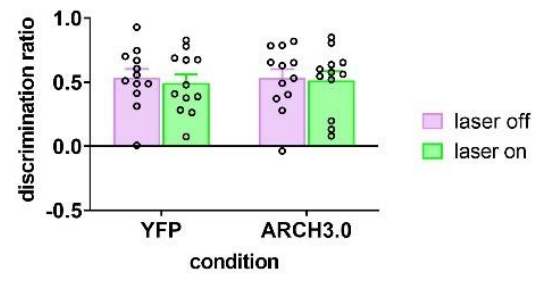

j.
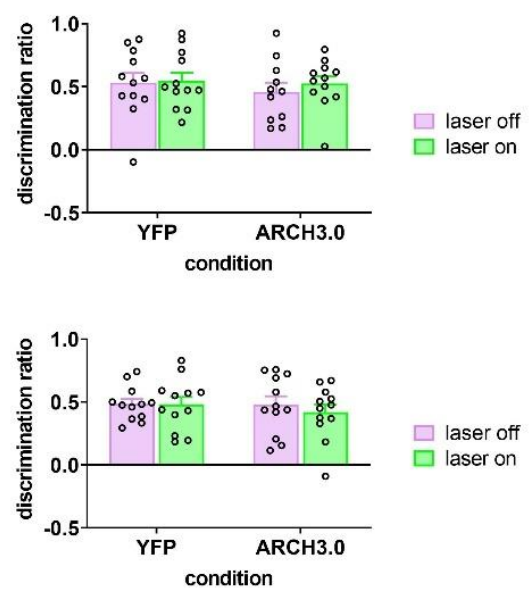

Supplementary Figure 3: Role of NRe to temporal order memory and contribution of reciprocal projections between NRe and $\mathrm{mPFC}$ on short term associative recognition memory and non-associative recognition memory. (a) Outline of the temporal order tasks, the delay between each sample phase and between sample phase 4 and test phase was either 5 minutes or 1 hour. (b) Performance in the temporal order memory task was significantly impaired at the $1 \mathrm{~h}$ delay but not the 5 minute delay after lesion of the NRe (two-way ANOVA lesion by delay $F(1,31)=4.249, p=0.048$; lesion $F(1,31)=7.890, p=0.009$; delay $F(1,33)=7.435, p=0.010,5$ 
min delay sham $n=9$, NRE lesion $n=9,1$ h delay sham $n=9$, NRE lesion $n=9$ ). ( c \& e) Outline of object-in-place memory task with a 5 -min delay between sample and test phase, to test memory encoding (c) laser light was delivered during the sample phase, to test memory retrieval (e) laser light was delivered during the test phase. (d) Deactivation of NRe $\rightarrow$ mPFC projections during memory encoding did not significantly alter performance in the object-inplace task when memory was tested at a 5 min delay (Two-way ANOVA virus by laser $F(1,21)$ $=0.455, p=0.507 ;$ virus $F(1,21)=0.183, p=0.673$; laser $F(1,21)=0.784, p=0.386$, YFP $n=12, A R C H$ $\mathrm{n}=11)$. (f) Deactivation of $\mathrm{mPFC} \rightarrow$ NRe projections during memory retrieval did not significantly alter performance in the object-in-place memory task when memory was tested at a 5 minute delay (two-way ANOVA virus by laser $F(1,22)=0.024, p=0.879$; virus $F(1,22)=0.021, p=0.887$; laser $F(1,22)=0.126, p=0.726$ ). ( $g$ \& i) Outline of the object recognition memory task based on the object-in-place task, laser light was delivered during the sample phase $(\mathrm{g})$ to test the effect on memory encoding, alternatively laser light was delivered during the sample phase (i) to test memory retrieval. (h) Deactivation of NRe $\rightarrow$ mPFC projections during memory encoding did not significantly alter object recognition memory performance (two-way ANOVA virus by laser $F(1,22)=0.148, p=0.704$; virus $F(1,22)=0.647, p=0.430$; laser $F(1,22)=0.207, p=0.653)$. (j) Deactivation of $\mathrm{mPFC} \rightarrow$ NRe projections during memory retrieval did not significantly alter object recognition memory performance (two-way ANOVA virus by laser $F(1,22)=0.017$, $p=0.896$; virus $F(1,22)=0.033, p=0.896$; laser $F(1,22)=0.618, p=0.440)$. YFP $n=12, A R C H n=12$ except where stated. Data presented as mean + sem (bar and error bar) and individual values (open circles). 
a. sample phase

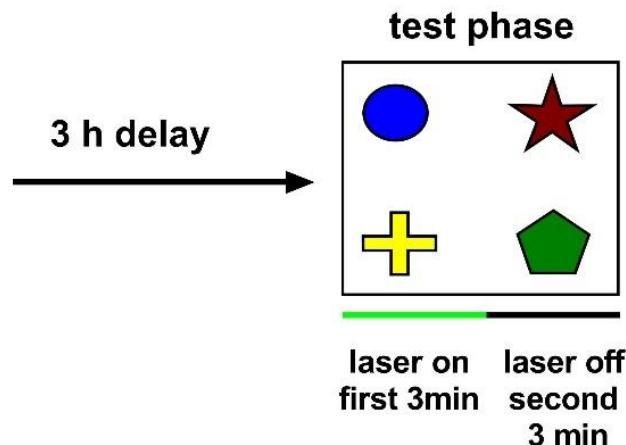

b.

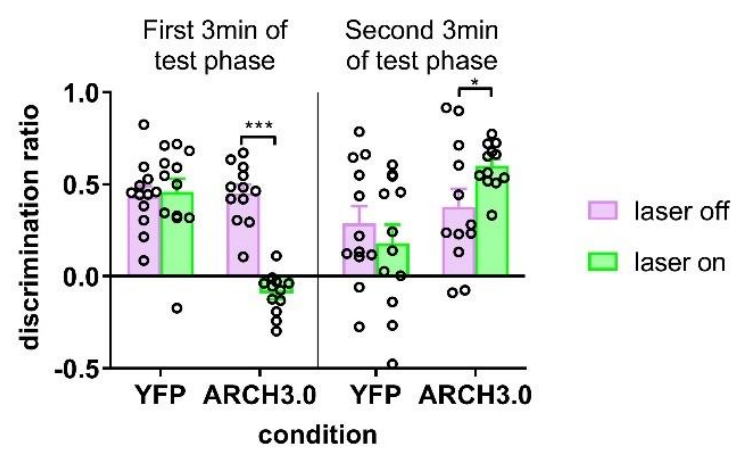

d.

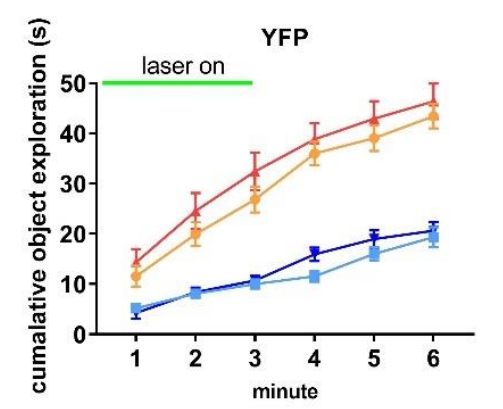

c.

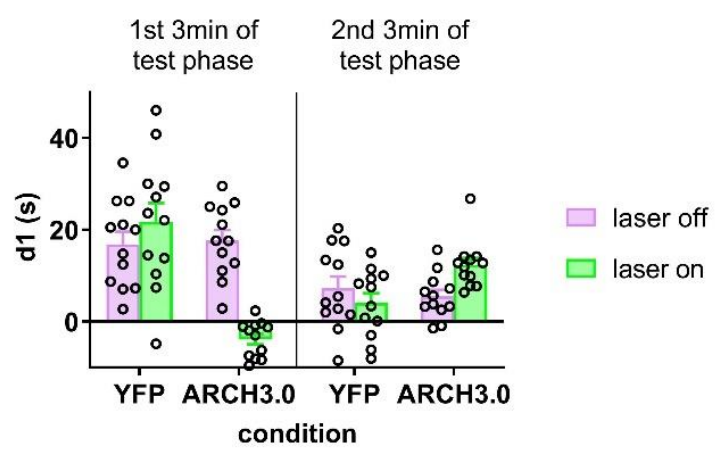

e.

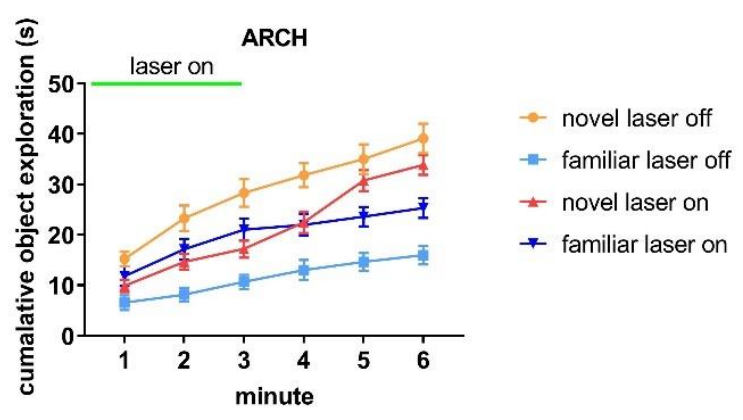

Supplementary Figure 4: Performance in the object-in-place task is restored when deactivation of mPFC $\rightarrow$ NRe projections is halted during the test phase. (a) Outline of object-in-place task, the test phase was extended to 6 minutes, laser light was delivered for the first 3 minutes of the test phase. (b $\&$ c) Object-in-place memory performance is impaired when mPFC $\rightarrow$ NRe projections are deactivated but is restored when deactivation ceases, this is observed when data is analysed as (b)discrimination ratio (three way-ANOVA virus by laser by period of test phase $F(1,22)=14.622, p=0.001$ ) or (c)discrimination index (D1) (three-way ANOVA virus by laser by period of test phase $F(1,22)=16.702$, $p=0.001$ ), data presented as mean + SEM. (d) Cumulative object exploration of the rearranged objects (novel) and non-rearranged objects (familiar) during the test phase in animals transfected with YFP. (e) Cumulative object exploration of the rearranged objects (novel) and non-rearranged objects (familiar) during the test phase in animals transfected with $A R C H$, data presented as mean \pm SEM, YFP $n=12$, ARCH $n=12, * p<0.05, * * p<0.01, * * * p<0.001$. 
a.

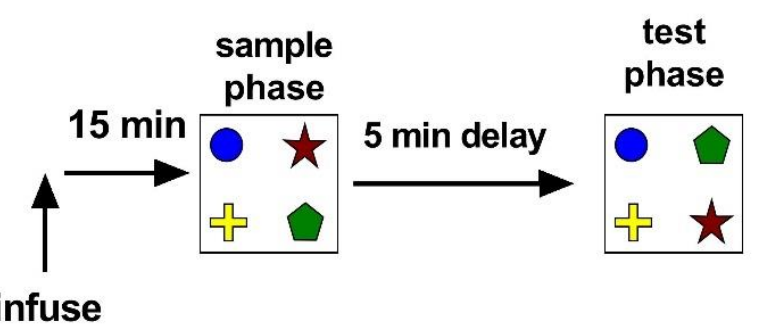

c.

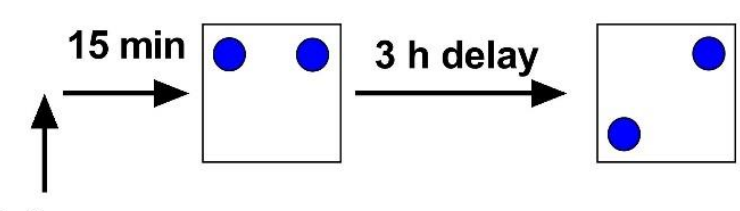

infuse

e.

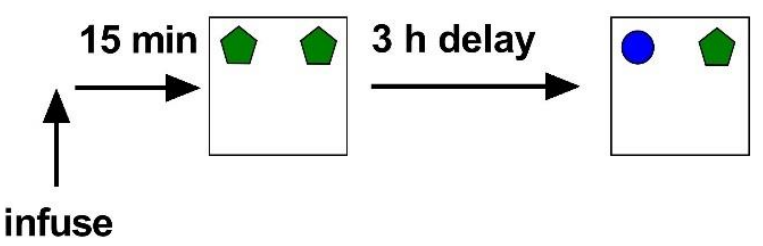

b.

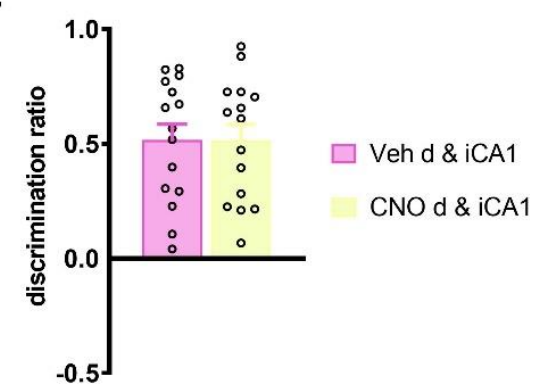

d.

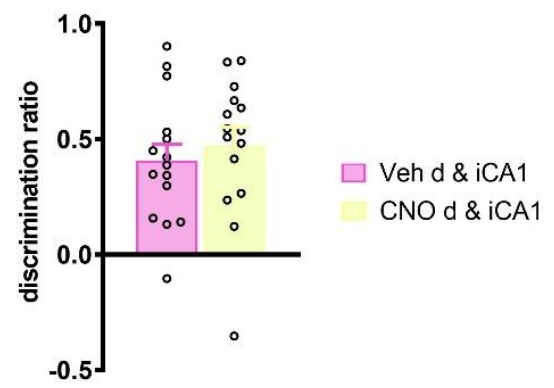

f.

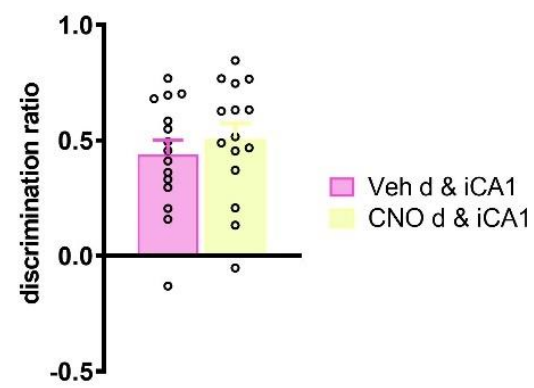

Supplementary Figure 5: Projections from NRe to CA1 region of hippocampus are not required for short term associative recognition memory or non-associative recognition memory. (a) Outline of the object-in-place task, CNO or vehicle was infused 15 minutes before the test phase into both dCA1 and iCA1, object-in-place memory was tested at a 5-min delay. (b) Deactivation of NRe $\rightarrow$ CA1 projections did not alter performance in the object-in-place memory task when memory was tested at a 5 minute delay (one-way ANOVA $F(1,14)=0.001, P=0.998$ ). f) Outline of object location task, CNO or vehicle was infused 15 min before the sample phase. (g) Deactivation of NRe $\rightarrow$ d\&iCA1 projections did not significantly alter object location memory performance (one-way ANOVA $F(1,14)=0.437$, $p=0.519$ ). (h) Outline of object recognition task, $C N O$ or vehicle was infused 15 minutes before the sample phase. (i) Deactivation of NRe $\rightarrow$ d\&iCA1 projections did not significantly alter object recognition memory performance (one-way ANOVA $F(1,14)=0.386, p=0.545$ ). Data presented as mean +sem (bar and error bar) and individual values (open circles), Veh $n=15, C N O n=15$. 